\title{
LAGRANGE IDENTITY IN LINEAR VISCOELASTICITY
}

\author{
S. CHIRITÃ \\ Mathematical Seminarium, University of Iaşi, 6600-Iaşi, Romania \\ S. RIONERO \\ Dipartimento di Matematica e sue Applicazioni, Università di Napoli, Via Mezzocannone 8, I-80134 \\ Napoli, Italy
}

(Communicated by E. SOOS)

\begin{abstract}
An approach based on the Lagrange identity is developed for the study of the initial boundary value problems of linear viscoelasticity. Estimations are obtained for describing the Liapunov stability and continuous data dependence of solutions. The main differences between the respective estimates consist in the measure of continuity and the constraint sets on which they are valid. The Lagrange identities are also used in order to obtain some reciprocal relations. The Cesaro means and autocorrelations of the velocity field and the strain field are introduced and it is proved that they exist and have a common value.
\end{abstract}

\section{INTRODUCTION}

The problem of stability of viscous motions has attracted in the past as nowadays many writers. Previous studies on uniqueness and continuous dependence in linear viscoelasticity have been based almost exclusively on the assumption that the initial (equilibrium) relaxation tensor is positive definite or strongly elliptic. Exceptions include a result of the paper by Brun [1], where an assumption concerning negative definiteness of the initial time derivative of the relaxation tensor is used; the study by Wilkes [2] uses the results of Gurtin and Herrera [3] on the dissipative materials; and, more recently, a paper by Rionero and Chiriţă [4] uses the consequences of the second law of thermodynamics established by Wilkes [5].

The present objective is to examine by means of a new approach the initial boundary value problems of linear viscoelasticity. The approach is developed on the basis of the Lagrange identity and its consequences.

It is shown that the total energy, consisting of the sum of the kinetical energy, the strain energy and the dissipation energy, is conserved. As a consequence of the local form of the Clausius-Duhem inequality, the dissipation energy is positive. When the strain energy is positive, a stability theorem is established by means of the Schwarz and Gronwall inequalities. And when the strain energy is of indefinite sign, the method of logarithmic convexity is modified to show that solutions are Hölder stable on all compact sub-intervals of the maximal interval of existence $[0, T)$. While the other consequences of the Lagrange identity lead in the first instance to continuity only on compact sub-intervals of the half interval $[0, T / 2)$ (see also [4]).

Further, we prove how a consequence of the Lagrange identity may be used in order to obtain the classical reciprocal relations established in [6]. We also indicate how the Lagrange identity and its consequences may be used in order to deduce new reciprocal theorems.

Finally, we consider a viscoelastic solid subjected to prescribed body force field; part of the boundary is clamped and the remainder is subjected to a prescribed traction. We suppose that the external force systems have an appropriate behavior when the time tends to infinity. Under the usual symmetry of relaxation tensor and positive definiteness of strain energy, we prove that the total energy tends to a finite limit as time tends to infinity, and, as a consequence the limit of dissipation energy exists and is finite. Furthermore, we prove that the autocorrelations of the velocity and strain fields exist and are equal, and the Cesàro mean kinetic and strain energies exist and are equally.

The plane of the paper is the following one. In Section 2 we set down the basic equations and initial and boundary conditions governing the solution to the problems of the linear theory of viscoelasticity. Some restrictions upon the relaxation tensor are discussed too. Section 3 
contains the derivation of some general consequences of the fundamental system of equations upon the viscoelastic processes. Section 4 examines the uniqueness of solutions under various assumptions upon the relaxation tensor. Section 5 is devoted to the treatment of the continuous dependence with respect to the data. A theorem is obtained for description the Liapunov stability of the solution. Reciprocal relations are obtained in Section 6. Section 7 concerns the autocorrelations of the velocity and strain fields. In the final Appendix we describe a system of Lagrange identities needed in the derivation of our results.

\section{LINEAR VISCOELASTICITY, PRELIMINARY CONSIDERATIONS}

For convenience, the notation and terminology chosen is almost identical to that of [7].

We consider a viscoelastic body $B$ with boundary $\partial B$ in the three-dimensional Euclidean point space. By an admissible state for $B$ we mean an ordered triplet $[\mathbf{u}, \mathbf{E}, \mathbf{S}]$ consisting of a vector displacement field $\mathbf{u}$ and symmetric tensor strain and stress fields $\mathbf{E}$ and $\mathbf{S}$ defined on $\bar{B}$. An admissible process for $B$ is an ordered triplet $[\mathbf{u}, \mathbf{E}, \mathbf{S}]$ whose value $[\mathbf{u}, \mathbf{E}, \mathbf{S}](t)$ is an admissible state at each time $t$. By an initial past history we mean a past history $[\boldsymbol{\omega}, \mathbf{R}, \mathbf{T}]$ whose values are admissible states for $B$. We say that $[\mathbf{u}, \mathbf{E}, \mathbf{S}]$ has initial past history $[\boldsymbol{\omega}, \mathbf{R}, \mathbf{T}]$ up to time $t=0$, if and only if, $[\mathbf{u}, \mathbf{E}, \mathbf{S}]$ satisfies the initial past-history condition

$$
[\mathbf{u}, \mathbf{E}, \mathbf{S}](\mathbf{x}, t)=[\mathbf{\omega}, \mathbf{R}, \mathbf{T}](\mathbf{x}, t), \quad \text { on } \quad \bar{B} \times(-\infty, 0) \text {. }
$$

Let $\partial B_{1}$ and $\partial B_{2}$ be two arbitrary subsets of $\partial B$ so that $\partial B_{1} \cup \partial B_{2}=\partial B$, and $\partial B_{1} \cap \partial B_{2}=$ $\varnothing$. Throughout this paper we assume given the following data: a time interval $[0, T)$, the relaxation tensor $\mathbf{G}$ on $\bar{B} \times(-\infty, \infty)$, the density field $\rho$ on $B$, body forces $\mathbf{b}$ on $B \times(0, T)$, surface displacements $\hat{\mathbf{u}}$ on $\partial B_{1} \times(0, T)$, the surface tractions $\hat{\mathbf{s}}$ on $\partial B_{2} \times(0, T)$ and the past history $[\boldsymbol{\omega}, \mathbf{R}, \mathbf{T}]$ on $\bar{B} \times(-\infty, 0)$.

By a dynamic viscoelastic process for $B$ corresponding to the relaxation tensor $\mathbf{G}$, the mass density $\rho$ and the body force $\mathbf{b}$ we mean an admissible process $[\mathbf{u}, \mathbf{E}, \mathbf{S}]$ for $B$, such that:

(a) the values of $\mathbf{u}, \mathbf{E}$, and $\mathbf{S}$ are continuous fields on $\bar{B}$;

(b) the displacement $\mathbf{u}$ is a process of class $C^{2}$ whose values are of class $C^{2}$ vector fields on $\bar{B}$

(c) on $B$, the processes $\mathbf{u}, \mathbf{E}$, and $\mathbf{S}$ satisfy the equation of motion

$$
\operatorname{div} \mathbf{S}+\rho \mathbf{b}=\rho \ddot{\mathbf{u}} \text {, }
$$

the constitutive equation

$$
\mathbf{S}(t)=\int_{-\infty}^{t} \mathbf{G}(t-z)[\dot{\mathbf{E}}(z)] \mathrm{d} z,
$$

and the strain-displacement relation

$$
\mathbf{E}=\frac{1}{2}\left(\nabla \mathbf{u}+\nabla \mathbf{u}^{T}\right)
$$

and the associated traction field $s$ is defined on $\partial B \times(-\infty, T)$ by

$$
\mathbf{s}=\mathbf{S n} \text {. }
$$

In the above relations we have used the following notations; div and $\nabla$ denote the divergence and gradient operators; superposed dots denote differentiation with respect to the time variable; $n$ is the outward unit normal to the boundary $\partial B$; the symbol ()$^{T}$ represents the transpose of () and $\bar{B}$ represents the closure of $B$; where no confusion may occur, we suppress the dependence upon the spatial variable. Further, we assume that for any pair of symmetric tensors $\mathbf{A}$ and $\mathbf{B}$

$$
\mathbf{A} \cdot \mathbf{G}[\mathbf{B}]=\mathbf{B} \cdot \mathbf{G}[\mathbf{A}]
$$

where the dot $(\cdot)$ denotes the appropriate inner product. Day [8] has shown that the relaxation tensor is symmetric if and only if the work done on every closed path starting from the virgin state is invariant under time-reversal.

By a solution of the dynamic viscoelastic boundary value problem corresponding to 
$\{\mathbf{G}, \rho, \mathbf{b} ; \hat{\mathbf{u}}, \hat{\mathbf{s}} ;[\boldsymbol{\omega}, \mathbf{R}, \mathbf{T}]\}$ we mean a dynamic viscoelastic process for $B,[\mathbf{u}, \mathbf{E}, \mathbf{S}]$, corresponding to $\{\mathbf{G}, \rho, b\}$ that satisfies the initial past-history condition (2.1) and the boundary conditions

$$
\begin{gathered}
\mathbf{u}(\mathbf{x}, t)=\hat{\mathbf{u}}(\mathbf{x}, t) \quad \text { on } \quad \partial B_{1} \times(0, T), \\
\mathbf{S}(\mathbf{x}, t) \mathbf{n}=\hat{\mathbf{s}}(\mathbf{x}, t)) \quad \text { on } \quad \partial B_{2} \times(0, T) .
\end{gathered}
$$

Under a lot of assumptions on $\mathbf{G}$, Dafermos $[9,10]$ showed the existence and asymptotic stability of the solution to the dynamic viscoelastic boundary value problem.

We say that $\Psi(\cdot)$ is of positive type if for every symmetric tensor $\mathbf{A}(\cdot)$ in $C^{0}$ on $(-\infty, \infty)$

$$
\pi_{\mathbf{A}}(t)=\int_{-\infty}^{t} \int_{-\infty}^{t} \mathbf{A}(z) \cdot \Psi(2 t-z-r)[\mathbf{A}(r)] \mathrm{d} z \mathrm{~d} r \geqslant 0 \text { for all } t \in(-\infty, \infty) .
$$

We say that $\Psi$ is of positive definite type if $\Psi$ is of positive type and $\pi_{A}(t)=0$ on $(-\infty, \infty)$ implies $\mathbf{A}(s)=\mathbf{0}$ on $(-\infty, \infty)$.

Remark. As it is shown by Wilkes [5], the local form of the Clausius-Duhem inequality implies

$$
-\int_{-\infty}^{t} \int_{-\infty}^{t} \dot{\mathbf{E}}(z) \cdot \dot{\mathbf{G}}(2 t-z-r)[\dot{\mathbf{E}}(r)] \mathrm{d} z \mathrm{~d} r \geqslant 0, \quad t \in(-\infty, \infty),
$$

so that the dissipation energy on $B \times[0, t]$,

$$
\mathscr{D}(t)=-\int_{0}^{t}\left\{\int_{-\infty}^{s} \int_{-\infty}^{s} \int_{B} \dot{\mathbf{E}}(z) \cdot \dot{\mathbf{G}}(2 s-z-r)[\dot{\mathbf{E}}(r)] \mathrm{d} V \mathrm{~d} z \mathrm{~d} r\right\} \mathrm{d} s,
$$

is positive. Therefore, $-\dot{\mathbf{G}}(\cdot)$ satisfies a condition of type defined by (2.9).

In fact, in the subsequent analysis we have need that $\mathbf{G}(\cdot)$ to be of positive type or positive definite type. As it is easily seen, the inequality (2.10) is satisfied, for instance, by any relaxation tensor $\mathbf{G}(\cdot)$ of the form

$$
\mathbf{G}(t)=\mathbf{G}_{0}+\sum_{i=1}^{n} \lambda_{i} \mathbf{G}_{i} \exp \left(-\mu_{i} t\right)
$$

where $\lambda_{i}$ and $\mu_{i}$ are positive constants, and $\mathbf{G}_{i}(i=1,2, \ldots, n)$ are positive tensor fields. Moreover, $\mathbf{G}(\cdot)$ is of positive type if $\mathbf{G}_{0}$ is a positive tensor field.

\section{SOME CONSEQUENCES ON VISCOELASTIC PROCESSES}

In this section, we outline some general consequences of the fundamental system of equations upon the viscoelastic processes.

Let $[\mathbf{u}, \mathbf{E}, \mathbf{S}]$ be a viscoelastic process corresponding to $\{\mathbf{G}, \rho, \mathbf{b} ; \hat{\mathbf{u}}, \hat{\mathbf{s}} ;[\boldsymbol{\omega}, \mathbf{R}, \mathbf{T}]\}$. Corresponding to this viscoelastic process we introduce the kinetic energy

$$
K(t)=\frac{1}{2} \int_{B} \rho \dot{u}(t) \cdot \dot{\mathbf{u}}(t) \mathrm{d} V,
$$

the strain energy $†$

$$
U(t)=\frac{1}{2} \int_{-\infty}^{t} \int_{-\infty}^{t} \int_{B} \dot{\mathbf{E}}(z) \cdot \mathbf{G}(2 t-z-r)[\dot{\mathbf{E}}(r)] \mathrm{d} V \mathrm{~d} z \mathrm{~d} r,
$$

† Staverman and Schwarzl [11], whose point of departure has been to regard the viscoelastic material as consisting of a network of linear viscous and elastic elements (i.e. dashpots and springs), pursuing the idea of model representation, arrived at the following expression for the free energy (per unit volume) of the solid

$$
\dot{F}_{1}=\frac{1}{2} \int_{-\infty}^{t} \int_{-\infty}^{t} G\left(2 t-\tau_{1}-\tau_{2}\right) \dot{\varepsilon}\left(\tau_{1}\right) \dot{\varepsilon}\left(\tau_{2}\right) \mathrm{d} \tau_{1} \mathrm{~d} \tau_{2}
$$

where $\dot{\varepsilon}(\tau)$ is the strain rate history to which the solid has been subjected in the interval $-\infty<t \leqslant t$, and $G(t)$ is the given relaxation modulus. Similar expressions are considered by Bland [12] and Hunter [13] (see also Breuer and Onat [14], Christensen and Naghdi [15], Eringen [16] and Dill [17]). 
the total energy

and

$$
\mathscr{E}(t)=K(t)+U(t)+\mathscr{D}(t)
$$

$$
\begin{gathered}
I(t)=\frac{1}{2} \int_{B} \rho \mathbf{u}(t) \cdot \mathbf{u}(t) \mathrm{d} V-\frac{1}{2} \int_{0}^{t}\left\{\int_{-\infty}^{s} \int_{-\infty}^{s} \int_{B} \mathbf{E}(z) \cdot \dot{\mathbf{G}}(2 s-z-r)[\mathbf{E}(r)] \mathrm{d} V \mathrm{~d} z \mathrm{~d} r\right\} \mathrm{d} s, \\
P(z, r)=\int_{B} \rho \dot{\mathbf{u}}(z) \cdot \mathbf{b}(r) \mathrm{d} V+\int_{\partial B} \dot{\mathbf{u}}(z) \cdot \mathbf{s}(r) \mathrm{d} A \\
W(z, r)=\int_{B} \rho \mathbf{u}(z) \cdot \mathbf{b}(r) \mathrm{d} V+\int_{\partial B} \mathbf{u}(z) \cdot \mathbf{s}(r) \mathrm{d} A
\end{gathered}
$$

Obviously, $P(t, t)$ and $W(t, t)$ represent the power and virtual work of the external loads. The function $I$ appears to have no universally accepted name but nonetheless it is useful in studies of viscoelastic stability.

We have now assembled all the preliminary material needed to derive the result of coupling of the identities (A1)-(A9) from the Appendix with the basic equations (2.2)-(2.5).

Lemma 1. Let $B$ be a regular region of space and let $\mathbf{G}(\cdot)$ be symmetric. Let $[\mathbf{u}, \mathbf{E}, \mathbf{S}]$ be a solution to the dynamic viscoelastic boundary value problem corresponding to $\{\mathbf{G}, \rho, \mathbf{b} ; \hat{\mathbf{u}}, \hat{\mathbf{s}} ;[\boldsymbol{\omega}, \mathbf{R}, \mathbf{T}]\}$. Then for each time $t \geqslant 0$, the following energy conservation law holds

$$
\mathscr{E}(t)=\mathscr{E}(0)+\int_{0}^{t} P(s, s) \mathrm{d} s, \quad t \geqslant 0 .
$$

Proof. We first set $H=L^{2}(B)$ and

$$
\mathbf{v}(t)=\mathbf{w}(t)=\mathbf{u}(t),
$$

into (A3). On the basis of the equation (2.2) and the relations $(2.3)-(2.6),(3.1),(3.5)$ and the divergence theorem we get

$$
K(t)=K(0)+\int_{0}^{t} P(s, s) \mathrm{d} s-\int_{0}^{t}\left\{\int_{B} \dot{\mathbf{E}}(s) \cdot \int_{-\infty}^{s} \mathbf{G}(s-z)[\dot{\mathbf{E}}(z)] \mathrm{d} z \mathrm{~d} V\right\} \mathrm{d} s .
$$

On the other hand, it is easy to see that (2.11) and (3.2) yield

$$
\int_{B} \dot{\mathbf{E}}(s) \cdot \int_{-\infty}^{s} \mathbf{G}(s-z)[\dot{\mathbf{E}}(z)] \mathrm{d} z \mathrm{~d} V=\frac{\mathrm{d} U}{\mathrm{~d} s}(s)+\frac{\mathrm{d} \mathscr{D}}{\mathrm{d} s}(s)
$$

Further, we substitute the relation (3.10) into (3.9) and use the notation of (3.1)-(3.5). This concludes the proof.

The following lemma is necessary to obtain Hölder continuous dependence of solutions.

LEMMA 2. Let $B$ be a regular region of space and let $\mathbf{G}(\cdot)$ be symmetric. Let $[\mathbf{u}, \mathbf{E}, \mathbf{S}]$ be a solution to the dynamic viscoelastic boundary value problem corresponding to $\{\mathbf{G}, \rho, \mathbf{b} ; \hat{\mathbf{u}}, \hat{\mathbf{s}} ;[\boldsymbol{\omega}, \mathbf{R}, \mathbf{T}]\}$. Then we have

$$
\begin{aligned}
\frac{\mathrm{d} I}{\mathrm{~d} t}(t)= & \int_{0}^{t}[4 K(s)+2 \mathscr{D}(s)] \mathrm{d} s+\frac{\mathrm{d} I}{\mathrm{~d} t}(0)-2 \mathscr{E}(0) t \\
& -2 \int_{0}^{t} \int_{0}^{s} P(z, z) \mathrm{d} z \mathrm{~d} s+\int_{0}^{t} W(s, s) \mathrm{d} s, \quad t \geqslant 0 .
\end{aligned}
$$

Proof. Firstly, we make use of the relation (3.8) into (A2) so that we get

$$
\int_{B} \rho \mathbf{u}(t) \cdot \dot{\mathbf{u}}(t) \mathrm{d} V=\int_{B} \rho \mathbf{u}(0) \cdot \dot{\mathbf{u}}(0) \mathrm{d} V+\int_{0}^{t} \int_{B} \rho[\dot{\mathbf{u}}(s) \cdot \dot{\mathbf{u}}(s)+\mathbf{u}(s) \cdot \ddot{\mathbf{u}}(s)] \mathrm{d} V \mathrm{~d} s .
$$


Further, we take into account the relations (2.2)-(2.6) and the divergence theorem in order to deduce

$$
\begin{aligned}
\int_{B} \rho \mathbf{u}(t) \cdot \dot{\mathbf{u}}(t) \mathrm{d} V= & \int_{B} \rho \mathbf{u}(0) \cdot \dot{\mathbf{u}}(0) \mathrm{d} V+2 \int_{0}^{t} K(s) \mathrm{d} s+\int_{0}^{t} W(s, s) \mathrm{d} s \\
& -\int_{0}^{t}\left\{\int_{B} \mathbf{E}(s) \cdot \int_{-\infty}^{s} \mathbf{G}(s-z)[\dot{\mathbf{E}}(z)] \mathrm{d} z \mathrm{~d} V\right\} \mathrm{d} s .
\end{aligned}
$$

A straightforward calculation proves that

$$
\begin{aligned}
\int_{B} \mathbf{E}(s) \cdot \int_{-\infty}^{s} \mathbf{G}(s-z) & {[\dot{\mathbf{E}}(z)] \mathrm{d} z \mathrm{~d} V } \\
= & 2 U(s)-\frac{\mathrm{d}}{\mathrm{d} s}\left\{\frac{1}{2} \int_{-\infty}^{s} \int_{-\infty}^{s} \int_{B} \mathbf{E}(z) \cdot \dot{\mathbf{G}}(2 s-z-r)[\mathbf{E}(r)] \mathrm{d} V \mathrm{~d} z \mathrm{~d} r\right\} .
\end{aligned}
$$

Therefore, the relations (3.1)-(3.4) and (3.13) and (3.14) imply

$$
\frac{\mathrm{d} I}{\mathrm{~d} t}(t)=\frac{\mathrm{d} I}{\mathrm{~d} t}(0)+2 \int_{0}^{t}[K(s)-U(s)] \mathrm{d} s+\int_{0}^{t} W(s, s) \mathrm{d} s .
$$

Finally, if we use the relations (3.1)-(3.3) and the identity (3.7) into (3.15) we easily obtain the identity (3.11). Thus, the proof is complete.

Lemma 3. Let $B$ be a regular region of space and let $\mathbf{G}(\cdot)$ be symmetric. Let $[\mathbf{u}, \mathbf{E}, \mathbf{S}]$ be a solution to the dynamic viscoelastic boundary value problem corresponding to $\{\mathbf{G}, \rho, \mathbf{b} ; \hat{\mathbf{u}}, \hat{\mathbf{s}} ;[\boldsymbol{\omega}, \mathbf{R}, \mathbf{T}]\}$. Then we have

$$
\begin{aligned}
\frac{\mathrm{d} I}{\mathrm{~d} t}(t)= & -\frac{1}{2} \int_{-\infty}^{0} \mathrm{~d} z \int_{-\infty}^{2 t} \mathrm{~d} r \int_{B} \mathbf{E}(z) \cdot \dot{\mathbf{G}}(2 t-z-r)[\mathbf{E}(r)] \mathrm{d} V \\
& +\frac{1}{2} \int_{B} \rho[\mathbf{u}(0) \cdot \dot{\mathbf{u}}(2 t)+\dot{\mathbf{u}}(0) \cdot \mathbf{u}(2 t)] \mathrm{d} V \\
& +\frac{1}{2} \int_{0}^{t}[W(t+s, t-s)-W(t-s, t+s)] \mathrm{d} s, \quad t \in\left[0, \frac{T}{2}\right) .
\end{aligned}
$$

Proof. If we use the relation (3.8) into (A4) and then we take into account the equations (2.2)-(2.5) and the divergence theorem, we deduce

$$
\begin{aligned}
& 2 \int_{B} \rho \mathbf{u}(t) \cdot \dot{\mathbf{u}}(t) \mathrm{d} V=\int_{B} \rho[\mathbf{u}(0) \cdot \dot{\mathbf{u}}(2 t)+\dot{\mathbf{u}}(0) \cdot \mathbf{u}(2 t)] \mathrm{d} V \\
& \quad+\int_{0}^{t}[W(t+s, t-s)-W(t-s, t+s)] \mathrm{d} s \\
& \quad+\int_{0}^{t} \int_{B}[\mathbf{E}(t-s) \cdot \mathbf{S}(t+s)-\mathbf{E}(t+s) \cdot \mathbf{S}(t-s)] \mathrm{d} V \mathrm{~d} s, \quad t \in\left[0, \frac{T}{2}\right) .
\end{aligned}
$$

In view of the relations (2.3) and (2.6) and by means of an integration by parts we deduce

$$
\begin{aligned}
\int_{B} & {[\mathbf{E}(t-s) \cdot \mathbf{S}(t+s)-\mathbf{E}(t+s) \cdot \mathbf{S}(t-s)] \mathrm{d} V } \\
= & \int_{B}\left\{\mathbf{E}(t-s) \cdot \int_{-\infty}^{t+s} \dot{\mathbf{G}}(t+s-z)[\mathbf{E}(z)] \mathrm{d} z\right. \\
& \left.-\mathbf{E}(t+s) \cdot \int_{-\infty}^{t-s} \dot{\mathbf{G}}(t-s-z)[\mathbf{E}(z)] \mathrm{d} z\right\} \mathrm{d} V \\
= & -\frac{\mathrm{d}}{\mathrm{d} s}\left\{\int_{-\infty}^{t-s} \mathrm{~d} s \int_{-\infty}^{t+s} \mathrm{~d} r \int_{B} \mathbf{E}(z) \cdot \dot{\mathbf{G}}(2 t-z-r)[\mathbf{E}(r)] \mathrm{d} V\right\} .
\end{aligned}
$$

If we substitute the relation (3.18) into (3.17) and use the notation of (3.4), then we find the identity (3.16). 
Lemma 4 . Let $B$ be a regular region of space and let $\mathbf{G}(\cdot)$ be symmetric. Let $[\mathbf{u}, \mathbf{E}, \mathbf{S}]$ be a solution to the dynamic viscoelastic boundary value problem corresponding to $\{\mathbf{G}, \rho, \mathbf{b} ; \hat{\mathbf{u}}, \hat{\mathbf{s}}$; $[\boldsymbol{\omega}, \mathbf{R}, \mathbf{T}]\}$. Then, we have

$$
\begin{aligned}
K(t)= & -\frac{1}{2} \mathscr{D}(t)+\frac{1}{2} \mathscr{E}(0)+\frac{1}{4} \int_{B} \rho \dot{\mathbf{u}}(0) \cdot \dot{\mathbf{u}}(2 t) \mathrm{d} V \\
& -\frac{1}{4} \int_{-\infty}^{0} \mathrm{~d} z \int_{-\infty}^{2 t} \mathrm{~d} r \int_{B} \dot{\mathbf{E}}(z) \cdot \mathbf{G}(2 t-z-r)[\dot{\mathbf{E}}(r)] \mathrm{d} V \\
& +\frac{1}{4} \int_{0}^{t}[P(t+s, t-s)+2 P(s, s)-P(t-s, t+s)] \mathrm{d} s, \quad t \in\left[0, \frac{T}{2}\right) \\
U(t)= & -\frac{1}{2} \mathscr{D}(t)+\frac{1}{2} \mathscr{E}(0)-\frac{1}{4} \int_{B} \rho \dot{\mathbf{u}}(0) \cdot \dot{\mathbf{u}}(2 t) \mathrm{d} V \\
& +\frac{1}{4} \int_{-\infty}^{0} \mathrm{~d} z \int_{-\infty}^{2 t} \mathrm{~d} r \int_{B} \dot{\mathbf{E}}(z) \cdot \mathbf{G}(2 t-z-r)[\dot{\mathbf{E}}(r)] \mathrm{d} V \\
& -\frac{1}{4} \int_{0}^{t}[P(t+s, t-s)-2 P(s, s)-P(t-s, t+s)] \mathrm{d} s, \quad t \in\left[0, \frac{T}{2}\right) .
\end{aligned}
$$

Proof. We note that, with the choice (3.8), and by using the relations (2.2)-(2.5), the identity (A6) becomes

$$
\begin{aligned}
\int_{B} \rho \dot{\mathbf{u}}(t) \cdot \dot{\mathbf{u}}(t) \mathrm{d} V= & \int_{B} \rho \dot{\mathbf{u}}(0) \cdot \dot{\mathbf{u}}(2 t) \mathrm{d} V \\
& +\int_{0}^{t}[P(t+s, t-s)-P(t-s, t+s)] \mathrm{d} s \\
& +\int_{0}^{t} \int_{B}\left\{\dot{\mathbf{E}}(t-s) \cdot \int_{-\infty}^{t+s} \mathbf{G}(t+s-z)[\dot{\mathbf{E}}(z)] \mathrm{d} z\right. \\
& \left.-\dot{\mathbf{E}}(t+s) \cdot \int_{-\infty}^{t-s} \mathbf{G}(t-s-z)[\dot{\mathbf{E}}(z)] \mathrm{d} z\right\} \mathrm{d} V \mathrm{~d} s .
\end{aligned}
$$

But a simple calculation gives

$$
\begin{array}{r}
\int_{B}\left\{\dot{\mathbf{E}}(t-s) \cdot \int_{-\infty}^{t+s} \mathbf{G}(t+s-z)[\dot{\mathbf{E}}(z)] \mathrm{d} z-\dot{\mathbf{E}}(t+s) \cdot \int_{-\infty}^{t-s} \mathbf{G}(t-s-z)[\dot{\mathbf{E}}(z)] \mathrm{d} z\right\} \mathrm{d} V \\
=-\frac{\mathrm{d}}{\mathrm{d} s}\left\{\int_{-\infty}^{t-s} \mathrm{~d} z \int_{-\infty}^{t+s} \mathrm{~d} r \int_{B} \dot{\mathbf{E}}(z) \cdot \mathbf{G}(2 t-z-r)[\dot{\mathbf{E}}(r)] \mathrm{d} V\right\},
\end{array}
$$

such that the substitution into (3.21) leads to

$$
\begin{aligned}
2 K(t)-2 U(t)= & \int_{B} \rho \dot{\mathbf{u}}(0) \cdot \dot{\mathbf{u}}(2 t) \mathrm{d} V-\int_{-\infty}^{0} \mathrm{~d} z \int_{-\infty}^{2 t} \mathrm{~d} r \int_{B} \dot{\mathbf{E}}(z) \cdot \mathbf{G}(2 t-z-r)[\dot{\mathbf{E}}(r)] \mathrm{d} V \\
& +\int_{0}^{t}[P(t+s, t-s)-P(t-s, t+s)] \mathrm{d} s .
\end{aligned}
$$

It is easy to see that a combination of the relations (3.7) and (3.23) leads to the identities (3.19) and (3.20). The proof is complete.

We remark that the last Lemma leads to a separation of energies in the sense described in [1].

\section{UNIQUENESS RESULTS}

We first observe that the conservation energy identity (3.7) leads to a counterpart of the classical uniqueness theorem of Neumann in linear elastodynamics. Thus, we state the following theorem. 
THEOREM 1. Suppose that $\rho>0$ and the relaxation tensor $\mathbf{G}(\cdot)$ is symmetric and of positive type, and that $-\mathbf{G}(\cdot)$ is of positive type. Then the initial boundary value problem associated to the linear viscoelastodynamics has at most one solution.

THEOREM 2. Suppose $\rho>0$ and that the relaxation tensor is symmetric and $-\dot{\mathbf{G}}(\cdot)$ is of positive type. Then the initial boundary value problem associated to the linear viscoelastodynamics has at most one solution.

Proof. Because of linearity it is clearly sufficient to show that the only solution of the dynamic viscoelastic boundary value problem for $B$ corresponding to $\{\mathbf{G}, \rho, \mathbf{0} ; \mathbf{0}, \mathbf{0} ;[0,0,0]\}$ is the following

$$
[\mathbf{u}, \mathbf{E}, \mathbf{S}]=[\mathbf{0 , 0}, \mathbf{0}] \text {. }
$$

This is clearly implied by $I(t)=0$ for all $t \in[0, T)$. Hence, we assume, as in [18], that there is an interval $0 \leqslant t_{1}<t<t_{2} \leqslant T$, on which, for example, $I(t)>0$. On this interval we show that the function

$$
G(t)=\ln I(t), \quad t_{1}<t<t_{2},
$$

is a convex function of $t$, i.e. we show

$$
I^{2} \frac{\mathrm{d}^{2} G}{\mathrm{~d} t^{2}}=I \frac{\mathrm{d}^{2} I}{\mathrm{~d} t^{2}}-\left(\frac{\mathrm{d} I}{\mathrm{~d} t}\right)^{2} \geqslant 0, \quad t_{1}<t<t_{2}
$$

Obviously, without loss of generality, we may take $I\left(t_{1}\right)=0$. Now, by means of the relations (3.4), (3.5), (3.6) and (3.11) and the hypotheses of theorem, we find

$$
\begin{aligned}
\frac{\mathrm{d} I}{\mathrm{~d} t}(t) & =\int_{B} \rho \mathbf{u}(t) \cdot \dot{\mathbf{u}}(t) \mathrm{d} V-\int_{0}^{t}\left\{\int_{-\infty}^{s} \int_{-\infty}^{s} \int_{B} \mathbf{E}(z) \cdot \dot{\mathbf{G}}(2 s-z-r)[\dot{\mathbf{E}}(r)] \mathrm{d} V \mathrm{~d} z \mathrm{~d} r\right\} \mathrm{d} s, \\
\frac{d^{2} I}{\mathrm{~d} t^{2}}(t) & =2 \int_{B} \rho \dot{\mathbf{u}}(t) \cdot \dot{\mathbf{u}}(t) \mathrm{d} V-2 \int_{0}^{t}\left\{\int_{-\infty}^{s} \int_{-\infty}^{s} \int_{B} \dot{\mathbf{E}}(z) \cdot \dot{\mathbf{G}}(2 s-z-r)[\dot{\mathbf{E}}(r)] \mathrm{d} V \mathrm{~d} z \mathrm{~d} r\right\} \mathrm{d} s,
\end{aligned}
$$

so that, by means of the Schwarz inequality, we deduce the inequality (4.3). Together with the continuity of $I(t)$ this implies, after integration,

$$
I(t) \leqslant\left[I\left(t_{2}\right)\right]^{t-t_{1} t_{2}-t_{1}}\left[I\left(t_{1}\right)\right]^{t_{2}-t / t_{2}-t_{1}}, \quad t_{1} \leqslant t \leqslant t_{2}
$$

But it has been assumed already that $I\left(t_{1}\right)=0$, so that (4.6) immediately shows that $I(t)=0$ for $t_{1} \leqslant t \leqslant t_{2}$, contrary to hypothesis. Hence, $I(t)=0$ for all $t<T$. Finally, since $\rho$ is strictly positive and $-\dot{G}(\cdot)$ is of positive type, we may conclude that (4.1) holds in $\bar{B} \times[0, T)$, and uniqueness is proved.

RemarK. The above theorem was proved in [4] by using the identity (3.16). It is easy to see that a similar argument may be used to prove the above theorem by means of the identity (3.19).

\section{STABILITY RESULTS}

We come now to a consideration of the stability of motions in linear viscoelasticity. A Liapunov stability theorem is established from the conservation law of energy (3.7) by means of the Gronwall's lemma and under the hypothesis that the strain energy is positive.

We also obtain a priori estimates describing continuous dependence of the solution with respect to body force, initial and boundary data. The approach based upon the identity (3.11) and the convexity arguments yield the Hölder continuity valid on all compact sub-intervals of the maximal interval of existence $[0, T)$, while the method based on the identity (3.16) leads in the first instance to continuity only on the half interval $[0, T / 2)$. However, this second method possesses a distinct advantage in the extension to weak solutions since it is unnecessary separately to postulate conservation of energy. 
Other estimates may be obtained by using the identities (3.19) and (3.20). The main differences between the respective estimates are to be found in the measure of continuity and the constraint sets on which they are valid.

We now proceed to obtain the results.

THEOREM 3. (Liapunov stability). Suppose that $\rho>0$ and that the relaxation tensor $\mathbf{G}(\cdot)$ is symmetric and that $\mathbf{G}(\cdot)$ and $-\dot{\mathbf{G}}(\cdot)$ are of positive type. Let $[\mathbf{u}, \mathbf{E}, \mathbf{S}]$ be a solution to the dynamic viscoelastic boundary value problem corresponding to $\{\mathbf{G}, \rho, \mathbf{b} ; \mathbf{0}, \mathbf{0} ;[\mathbf{\omega}, \mathbf{R}, \mathbf{T}]\}$. Then

$$
\mathscr{E}(t)^{1 / 2} \leqslant \mathscr{E}(0)^{1 / 2}+\frac{1}{\sqrt{2}} \int_{0}^{t}\|\mathbf{b}(s)\| \mathrm{d} s, \quad t \in[0, T),
$$

where

$$
\|\mathbf{b}(s)\|^{2}=\int_{B} \rho|\mathbf{b}(s)|^{2} \mathrm{~d} V, \quad|\mathbf{b}(s)|^{2}=\mathbf{b}(s) \cdot \mathbf{b}(s) .
$$

Proof. In view of the boundary conditions, from the identity (3.7) we obtain, by means of the Schwarz's incquality and the inequality $K(t) \leqslant \mathscr{E}(t)$, that

$$
\mathscr{E}(t) \leqslant \mathscr{E}(0)+\sqrt{2} \int_{0}^{t} \mathscr{E}(s)^{1 / 2}\|\mathbf{b}(s)\| \mathrm{d} s, \quad t \in[0, T) .
$$

Therefore, the Gronwall's lemma [19] applied to Gronwall-type inequality (5.3) gives the estimate (5.1).

We next discuss the method based on the identity (3.11). We obtain estimates of sort given by Knops and Payne [20] by means of logarithmic convexity for linear elasticity.

Theorem 4. (Continuous dependence upon body force). Suppose that $\rho>0$ and the relaxation tensor $\mathbf{G}(\cdot)$ is symmetric and $-\dot{\mathbf{G}}(\cdot)$ is of positive type. Let $[\mathbf{u}, \mathbf{E}, \mathbf{S}]$ be a solution to the dynamic viscoelastic boundary value problem corresponding to $\{\mathbf{G}, \rho, \mathbf{b} ; \mathbf{0}, \mathbf{0} ;[\mathbf{0}, \mathbf{0}, \mathbf{0}]\}$, in the constraint set for which there exists $T_{1} \in[0, T)$ so that

$$
\int_{0}^{T_{1}} I(t) \mathrm{d} t \leqslant M_{1}^{2}, \quad M_{1}-\text { positive constant. }
$$

If

$$
F(t)=\int_{0}^{t} I(s) \mathrm{d} s+2 T_{1}^{4} \int_{0}^{T_{1}}\|\mathbf{b}(s)\|^{2} \mathrm{~d} s, \quad t \in\left[0, T_{1}\right]
$$

and

$$
F(t) \leqslant \mathrm{e}^{\delta(1-\delta)}\left[F\left(T_{1}\right)\right]^{\delta}[F(0)]^{1-\delta}, \quad \delta=t / T_{1}, \quad t \in\left[0, T_{1}\right] .
$$

Proof. In view of the hypotheses of the theorem, we have

$$
I(0)=\dot{I}(0)=\mathscr{E}(0)=0 .
$$

Obviously, by means of (3.4) and (5.7), from (5.5) and (3.11), we get

$$
\begin{aligned}
\dot{F}(t)=I(t)=\int_{0}^{t} \dot{I}(s) \mathrm{d} s & =\int_{0}^{t} \int_{B} \rho \mathbf{u}(s) \cdot \dot{\mathbf{u}}(s) \mathrm{d} V \mathrm{~d} s \\
& -\int_{0}^{t} \int_{0}^{s}\left\{\int_{-\infty}^{\eta} \int_{-\infty}^{\eta} \int_{B} \mathbf{E}(z) \cdot \dot{\mathbf{G}}(2 \eta-z-r)[\dot{\mathbf{E}}(r)] \mathrm{d} V \mathrm{~d} z \mathrm{~d} r\right\} \mathrm{d} \eta \mathrm{d} s,
\end{aligned}
$$

and

$$
\begin{aligned}
\ddot{F}(t)= & \dot{I}(t)=2 \int_{0}^{t} \int_{B} \rho \dot{\mathbf{u}}(s) \cdot \dot{\mathbf{u}}(s) \mathrm{d} V \mathrm{~d} s \\
& -2 \int_{0}^{t} \int_{0}^{s}\left\{\int_{-\infty}^{\eta} \int_{-\infty}^{\eta} \int_{B} \dot{\mathbf{E}}(z) \cdot \dot{\mathbf{G}}(2 \eta-z-r)[\dot{\mathbf{E}}(r)] \mathrm{d} V \mathrm{~d} z \mathrm{~d} r\right\} \mathrm{d} \eta \mathrm{d} s \\
& +\int_{0}^{t} \int_{B} \rho[\mathbf{u}(s) \cdot \mathbf{b}(s)-2(t-s) \dot{\mathbf{u}}(s) \cdot \mathbf{b}(s)] \mathrm{d} V \mathrm{~d} s .
\end{aligned}
$$


Therefore, from (5.5), (5.8), (5.9) and the Schwarz's inequality, we deduce

$$
\begin{aligned}
& F \ddot{F}-\dot{F}^{2} \geqslant 4 T_{1}^{4}\left(\int_{0}^{T_{1}}\|\mathbf{b}(s)\|^{2} \mathrm{~d} s\right)\left(\int_{0}^{t}\|\dot{\mathrm{u}}(s)\|^{2} \mathrm{~d} s\right) \\
&+F \int_{0}^{t} \int_{B} \rho[\mathbf{u}(s) \cdot \mathbf{b}(s)-2(t-s) \dot{\mathbf{u}}(s) \cdot \mathbf{b}(s)] \mathrm{d} V \mathrm{~d} s \\
&-F \int_{0}^{t} \int_{B} \rho \mathbf{u}(s) \cdot \mathbf{b}(s) \mathrm{d} V \mathrm{~d} s \leqslant T_{1}^{-2} F^{2} \\
& 2 F \int_{0}^{t} \int_{B}(t-s) \rho \dot{\mathbf{u}}(s) \cdot \mathbf{b}(s) \mathrm{d} V \mathrm{~d} s \leqslant T_{1}^{-2} F^{2} \\
&+T_{1}^{4}\left(\int_{0}^{T_{1}}\|\mathbf{b}(s)\|^{2} \mathrm{~d} s\right)\left(\int_{0}^{t}\|\dot{\mathbf{u}}(s)\|^{2} \mathrm{~d} s\right) .
\end{aligned}
$$

We are thus led to the inequality

$$
F \ddot{F}-\dot{F}^{2} \geqslant-2 T_{1}^{-2} F^{2} .
$$

Since $F(t)$ is strictly positive unless $\mathbf{b}(s)=0$ (except possibly on a set of measure zero), we may divide (5.11) by $F^{2}$ to obtain

$$
\frac{\mathrm{d}^{2}}{\mathrm{~d} t^{2}}[\ln F(t)] \geqslant-2 T_{1}^{-2}
$$

which yields the inequality (5.6). Thus, the proof is complete.

REMARK. Let $[\mathbf{u}, \mathbf{E}, \mathbf{S}]$ be a solution to the dynamic viscoelastic boundary value problem corresponding to $\{\mathbf{G}, \rho, \mathbf{0} ; \hat{\mathbf{u}}, \hat{\mathbf{s}} ;[\mathbf{0}, \mathbf{0}, 0]\}$, in the constraint set for which there exist $T_{1} \in[0, T)$ and the positive constants $M_{1}, M_{2}, M_{3}$ and $M_{4}$ so that the condition (5.4) is satisfied and, moreover, we have

$$
\int_{0}^{T_{1}} \int_{\partial B_{1}}|\mathbf{S}(s)|^{2} \mathrm{~d} A \mathrm{~d} s \leqslant M_{2}^{2}, \quad \int_{0}^{T_{1}} \int_{\partial B_{2}}|\mathbf{u}(s)|^{2} \mathrm{~d} A \mathrm{~d} s \leqslant M_{3}^{2}, \quad \int_{0}^{T_{1}} \int_{\partial B_{2}}|\dot{\mathbf{u}}(s)|^{2} \mathrm{~d} A \mathrm{~d} s \leqslant M_{4}^{2} .
$$

In this class, the continuous dependence upon the boundary data is described by the estimate

$$
F_{*}(t) \leqslant \mathrm{e}^{\delta(1-\delta)}\left[F_{*}\left(T_{1}\right)\right]^{\delta}\left[F_{*}(0)\right]^{1-\delta}, \quad \delta=t / T_{1}, \quad t \in\left[0, T_{1}\right],
$$

where

$$
\begin{aligned}
F_{*}(t)= & \int_{0}^{t} I(s) \mathrm{d} s+\frac{1}{2} T_{1}^{2}\left[M_{2}\left(\int_{0}^{T_{1}} \int_{\partial B_{1}}|\hat{\mathrm{u}}(s)|^{2} \mathrm{~d} A \mathrm{~d} s\right)^{1 / 2}+2 T_{1} M_{2}\left(\int_{0}^{T_{1}} \int_{\partial B_{1}}|\hat{\mathrm{u}}(s)|^{2} \mathrm{~d} A \mathrm{~d} s\right)^{1 / 2}\right. \\
& \left.+\left(2 T_{1} M_{4}+M_{3}\right)\left(\int_{0}^{T_{1}} \int_{\partial B_{2}}|\hat{\mathbf{s}}(z)|^{2} \mathrm{~d} A \mathrm{~d} z\right)^{1 / 2}\right] .
\end{aligned}
$$

THEOREM 5. (Continuous dependence upon initial data.) Suppose that $\rho>0$ and the relaxation tensor $\mathbf{G}(\cdot)$ is symmetric and $-\dot{\mathbf{G}}(\cdot)$ is of positive type. Let $[\mathbf{u}, \mathbf{E}, \mathbf{S}]$ be a solution to the dynamic viscoelastic boundary value problem corresponding to $\{\mathbf{G}, \rho, 0 ; 0,0 ;[\omega, R, T]\}$ in the class $\mathcal{M}$ of the viscoelastic processes for which

$$
I(t) \leqslant M, \quad t \in\left[0, T_{1}\right], \quad M \text {-positive constant. }
$$

Then, for initial data with initial total energy $\mathscr{E}(0) \leqslant 0$, we have

$$
\begin{gathered}
J(t) \leqslant J\left(T_{1}\right)^{\delta} J(0)^{1-\delta}, \quad \delta=t / T_{1}, \quad t \in\left[0, T_{1}\right], \\
J(t)=I(t)-\frac{1}{2}\left(T_{1}-t\right) \int_{-\infty}^{0} \int_{-\infty}^{0} \int_{B} \mathbf{E}(z) \cdot \dot{\mathbf{G}}(-z-r)[\mathbf{E}(r)] \mathrm{d} V \mathrm{~d} z \mathrm{~d} r
\end{gathered}
$$


and, for initial data with $\mathscr{E}(0)>0$, we have

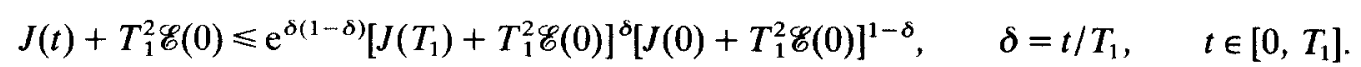

ProOF. We first note that a straightforward calculation gives

$$
\begin{aligned}
\frac{\mathrm{d}}{\mathrm{d} t}\left\{\frac{1}{2} \int_{-\infty}^{t} \int_{-\infty}^{t} \int_{B} \mathbf{E}(z) \cdot \dot{\mathbf{G}}(2 t-z-r)[\boldsymbol{E}(r)] \mathrm{d} V \mathrm{~d} z \mathrm{~d} r\right\} \\
=\int_{-\infty}^{t} \int_{-\infty}^{t} \int_{B} \mathbf{E}(z) \cdot \dot{\mathbf{G}}(2 t-z-r)[\dot{\mathbf{E}}(r)] \mathrm{d} V \mathrm{~d} z \mathrm{~d} r .
\end{aligned}
$$

Further, since $-\dot{\mathbf{G}}(\cdot)$ is of positive type, from (2.10), (3.4) and (5.18), we get

$$
J(t) \geqslant \frac{1}{2} \int_{B} \rho \mathbf{u}(t) \cdot \mathbf{u}(t) \mathrm{d} V-\frac{1}{2} \int_{0}^{t}\left\{\int_{-\infty}^{s} \int_{-\infty}^{s} \int_{B} \mathbf{E}(z) \cdot \dot{\mathbf{G}}(2 s-z-r)[\mathbf{E}(r)] \mathrm{d} V \mathrm{~d} z \mathrm{~d} r\right\} \mathrm{d} s .
$$

Moreover, from (5.18) and (5.20), we deduce

$$
\dot{J}(t)=\int_{B} \rho \mathbf{u}(t) \cdot \dot{\mathbf{u}}(t) \mathrm{d} V-\int_{0}^{t}\left\{\int_{-\infty}^{s} \int_{-\infty}^{s} \int_{B} \mathbf{E}(z) \cdot \dot{\mathbf{G}}(2 s-z-r)[\dot{\mathbf{E}}(r)] \mathrm{d} V \mathrm{~d} z \mathrm{~d} r\right\} \mathrm{d} s .
$$

Finally, from the identity (3.11), we deduce

$$
\ddot{J}(t)=2 \int_{B} \rho \dot{\mathbf{u}}(t) \cdot \dot{\mathbf{u}}(t) \mathrm{d} V-2 \int_{0}^{t}\left\{\int_{-\infty}^{s} \int_{-\infty}^{s} \int_{B} \dot{\mathbf{E}}(z) \cdot \dot{\mathbf{G}}(2 s-z-r)[\dot{\mathbf{E}}(r)] \mathrm{d} V \mathrm{~d} z \mathrm{~d} r\right\} \mathrm{d} s-2 \mathscr{E}(0) .
$$

Now, by Schwarz's inequality, from (5.21)-(5.23), we get

$$
J \ddot{J}-\dot{J}^{2} \geqslant-2 \mathscr{E}(0) J, \quad t \in\left[0, T_{1}\right]
$$

Further, the discussion cannot be conducted simultaneously for all types of initial data, however, so we separate accordingly the situations in which the initial total energy is respectively negative, zero or positive.

We begin by proving the Hölder stability of the solution assuming firstly that $\mathscr{E}(0) \leqslant 0$. By hypothesis $J(t)>0, t \in\left[0, T_{1}\right]$, and therefore, the inequality (5.24) may be immediately rewritten as

$$
\frac{\mathrm{d}^{2}}{\mathrm{~d} t^{2}}[\ln J(t)] \geqslant 0
$$

By Jensen's inequality, we obtain from (5.25) the inequality (5.17). Thus, the inequality (5.17) establishes that for processes of class $\mu$ the solution is Hölder stable on compact sub-intervals of $[0, T)$ in the norm $J$ for initial data with non-positive total energy.

When the initial data produces a positive total energy, we use a similar argument applied to the function, $J(t)+T_{1}^{2} \mathscr{E}(0)$. The previous manipulations then result in the inequality (5.19), so that now the solution is Hölder stable on compact sub-intervals of $[0, T)$ in the norm $J(t)+T_{1}^{2} \mathscr{E}(0)$ for initial data with positive total energy. Thus, the proof is complete.

Continuous dependence based on the identity (3.16) was discussed in [4]. Here, we indicate a new way to establish continuous dependence upon initial data. Therefore, we introduce the class of viscoelastic processes for which there exist $T_{1} \in[0, T)$ and the positive constants $M_{5}$ and $M_{6}$ so that

$$
\begin{gathered}
-\frac{1}{2} \int_{-\infty}^{t} \int_{-\infty}^{t} \int_{B} \mathbf{E}(z) \cdot \dot{\mathbf{G}}(2 t-z-r)[\mathbf{E}(r)] \mathrm{d} V \mathrm{~d} z \mathrm{~d} r \leqslant M_{5}^{2}, \quad t \in\left[0, T_{1}\right], \\
\int_{B} \rho \mathbf{u}(t) \cdot \mathbf{u}(t) \mathrm{d} V \leqslant M_{6}^{2}, \quad t \in\left[0, T_{1}\right]
\end{gathered}
$$


THEOREM 6. Suppose that $\rho>0$ and the relaxation tensor $\mathbf{G}(\cdot)$ is symmetric and $-\dot{\mathbf{G}}(\cdot)$ is of positive type. Let $[\mathbf{u}, \mathbf{E}, \mathbf{S}]$ be a solution to the dynamic viscoelastic boundary value problem corresponding to $\{\mathbf{G}, \rho, 0 ; 0,0 ;[\omega, \mathbf{K}, \mathbf{T}]\}$ in the class of the viscoelastic processes for which (5.26) and (5.27) hold. Then we have

$$
\begin{aligned}
I(t) \leqslant & \frac{1}{4}\|\mathbf{u}(0)\|^{2}+\frac{1}{4} M_{6}\|\mathbf{u}(0)\|+\frac{1}{4} T_{1} M_{6}\|\dot{\mathbf{u}}(0)\| \\
& +\frac{1}{2} T_{1} M_{5}\left(-\frac{1}{2} \int_{-\infty}^{0} \int_{-\infty}^{0} \int_{B} \mathbf{E}(z) \cdot \dot{\mathbf{G}}(-z-r)[\mathbf{E}(r)] \mathrm{d} V \mathrm{~d} z \mathrm{~d} r\right)^{1 / 2}, \quad t \in\left[0, \frac{T_{1}}{2}\right] .
\end{aligned}
$$

Proof. In view of the hypotheses of the theorem, from (3.16) we deduce

$$
\begin{aligned}
\frac{\mathrm{d} I}{\mathrm{~d} t}(t)= & -\frac{1}{2} \int_{-\infty}^{0} \int_{-\infty}^{0} \int_{B} \mathbf{E}(z) \cdot \dot{\mathbf{G}}(-z-r)[\mathbf{E}(r+2 t)] \mathrm{d} V \mathrm{~d} z \mathrm{~d} r \\
& +\frac{1}{2} \int_{B} \rho[\mathbf{u}(0) \cdot \dot{\mathbf{u}}(2 t)+\dot{\mathbf{u}}(0) \cdot \mathbf{u}(2 t)] \mathrm{d} V, \quad t \in\left[0, \frac{T}{2}\right) .
\end{aligned}
$$

Since $-\dot{\mathbf{G}}(\cdot)$ is of positive type, we have

$$
\begin{aligned}
- & \frac{1}{2} \int_{-\infty}^{0} \int_{-\infty}^{0} \int_{B} \mathbf{E}(s) \cdot \dot{\mathbf{G}}(-z-r)[\mathbf{E}(r+2 t)] \mathrm{d} V \mathrm{~d} z \mathrm{~d} r \\
& \leqslant\left\{-\frac{1}{2} \int_{-\infty}^{0} \int_{-\infty}^{0} \int_{B} \mathbf{E}(z) \cdot \dot{\mathbf{G}}(-z-r)[\mathbf{E}(r)] \mathrm{d} V \mathrm{~d} z \mathrm{~d} r\right\}^{1 / 2} \\
& \cdot\left\{-\frac{1}{2} \int_{-\infty}^{2 t} \int_{-\infty}^{2 t} \int_{B} \mathbf{E}(z) \cdot \dot{\mathbf{G}}(4 t-z-r)[\mathbf{E}(r)] \mathrm{d} V \mathrm{~d} z \mathrm{~d} r\right\}^{1 / 2} \\
& \leqslant M_{5}\left\{-\frac{1}{2} \int_{-\infty}^{0} \int_{-\infty}^{0} \int_{B} \mathbf{E}(z) \cdot \dot{\mathbf{G}}(-z-r)[\mathbf{E}(r)] \mathrm{d} V \mathrm{~d} z \mathrm{~d} r\right\}^{1 / 2}, \quad t \in\left[0, \frac{T_{1}}{2}\right] .
\end{aligned}
$$

An integration over $(0, s), 0<2 s \leqslant T_{1}$, gives

$$
\begin{aligned}
I(s) \leqslant & I(0)+\frac{1}{2} T_{1} M_{S}\left(-\frac{1}{2} \int_{-\infty}^{0} \int_{-\infty}^{0} \int_{B} \mathbf{E}(z) \cdot \dot{\mathbf{G}}(-z-r)[\mathbf{E}(r)] \mathrm{d} V \mathrm{~d} z \mathrm{~d} r\right)^{1 / 2} \\
& +\frac{1}{4} \int_{B} \rho[\mathbf{u}(0) \cdot \mathbf{u}(2 s)-\mathbf{u}(0) \cdot \mathbf{u}(0)] \mathrm{d} V+\frac{1}{2} \int_{0}^{s} \int_{B} \rho \dot{\mathbf{u}}(0) \cdot \mathbf{u}(2 t) \mathrm{d} V \mathrm{~d} t .
\end{aligned}
$$

Further, the inequality (5.28) follows from (5.31) by means of the Schwarz's inequality.

Remark. Alternatively, we may constrain the solution to lie in the set defined by (5.26) and

$$
\int_{0}^{T_{1}}\|\mathrm{u}(s)\|^{2} \mathrm{~d} s \leqslant M_{7}^{2}, \quad M_{T} \text { - positive constant. }
$$

Then, inequality (5.31) becomes after an integration over $(0, t), t \in\left[0, T_{1} / 2\right]$,

$$
\begin{aligned}
\int_{0}^{t} I(s) \mathrm{d} s \leqslant & \frac{1}{4} t\|\mathbf{u}(0)\|^{2}+\frac{1}{2} t T_{1} M_{5}\left(-\frac{1}{2} \int_{-\infty}^{0} \int_{-\infty}^{0} \int_{B} \mathbf{E}(z) \cdot \dot{\mathbf{G}}(-z-r)[\mathbf{E}(r)] \mathrm{d} V \mathrm{~d} z \mathrm{~d} r\right)^{1 / 2} \\
& +\frac{1}{4} \int_{0}^{t} \int_{B} \rho \mathbf{u}(0) \cdot \mathbf{u}(2 s) \mathrm{d} V \mathrm{~d} s+\frac{1}{2} \int_{0}^{t} \int_{B}(t-s) \rho \mathbf{u}(0) \cdot \mathbf{u}(2 s) \mathrm{d} V \mathrm{~d} s .
\end{aligned}
$$

By means of the Schwarz's inequality and the relation (5.32) we get

$$
\begin{aligned}
\int_{0}^{t} I(s) \mathrm{d} s \leqslant & \frac{1}{8} T_{1}\|\mathbf{u}(0)\|^{2}+\frac{1}{4} T_{1}^{2} M_{5}\left(-\frac{1}{2} \int_{-\infty}^{0} \int_{-\infty}^{0} \int_{B} \mathbf{E}(z) \cdot \dot{\mathbf{G}}(-z-r)[\mathbf{E}(r)] \mathrm{d} V \mathrm{~d} z \mathrm{~d} r\right)^{1 / 2} \\
& +\frac{1}{8} T_{1}^{1 / 2} M_{7}\|\mathbf{u}(0)\|+\frac{1}{8} T_{1}^{3 / 2} M_{7}\|\dot{\mathbf{u}}(0)\|, \quad t \in\left[0, \frac{T_{1}}{2}\right]
\end{aligned}
$$

The last inequality provides a second estimate for continuous dependence on initial data. 
Obviously, the inequalities (5.28) and (5.34) lead in the first instance to continuity only on the half interval. One can extend the interval of continuous dependence by using one of the methods described in [21].

\section{RECIPROCAL RELATIONS}

In the remainder of this paper we shall assume that $T$ is infinity. We show how the identities (A1)-(A9) may be used in order to obtain reciprocal relations. The approach based on the identity (A7) leads to the classical reciprocal relations established in [6]; while the method based on the identity (A4) leads to the reciprocal relations estalished in [4].

Let $f_{1}$ and $g_{1}$ be scalar fields continuous in time. We denote by $f_{1} * g_{1}$ the convolution of $f_{1}$ and $g_{1}$

$$
\left(f_{1} * g_{1}\right)(\mathbf{x}, t)=\int_{0}^{t} f_{1}(\mathbf{x}, t-s) g_{1}(\mathbf{x}, s) \mathrm{d} s
$$

We also introduce the functions

$$
l(t)=1, \quad g(t)=(l * l)(t)=t, \quad t \in[0, \infty) .
$$

Theorem 7. Let $B$ be a regular region of space and let $\mathbf{G}(\cdot)$ be symmetric. Let $\left[\mathbf{u}_{\alpha}, \mathbf{E}_{\alpha}, \mathbf{S}_{\alpha}\right]$, $(\alpha=1,2)$, be the solutions to the dynamic viscoelastic boundary value problem for $B$, corresponding to $\left\{\mathbf{G}, \rho, \mathbf{b}_{\alpha} ; \hat{\mathbf{u}}_{\alpha}, \hat{\mathbf{s}}_{\boldsymbol{\alpha}} ;\left[\boldsymbol{\omega}_{\alpha}, \mathbf{R}_{\alpha}, \mathbf{T}_{\alpha}\right]\right\},(\alpha=1,2)$, respectively. Then for each time $t \geqslant 0$,

$$
\begin{gathered}
\int_{B} \rho \mathbf{u}_{1} * \mathbf{b}_{2} \mathrm{~d} V+\int_{\partial B} \mathbf{u}_{1} * \mathbf{s}_{2} \mathrm{~d} A-\int_{B} \mathbf{E}_{1} * \mathbf{T}_{2}^{0} \mathrm{~d} V+\int_{B} \rho\left[\mathbf{u}_{1}(t) \cdot \dot{\mathbf{u}}_{2}(0)+\dot{\mathbf{u}}_{1}(t) \cdot \mathbf{u}_{2}(0)\right] \mathrm{d} V \\
=\int_{B} \rho \mathbf{u}_{2} * \mathbf{b}_{1} \mathrm{~d} V+\int_{\partial B} \mathbf{u}_{2} * \mathbf{s}_{1} \mathrm{~d} A-\int_{B} \mathbf{E}_{2} * \mathbf{T}_{1}^{0} \mathrm{~d} V+\int_{B} \rho\left[\mathbf{u}_{1}(0) \cdot \dot{\mathbf{u}}_{2}(t)+\dot{\mathbf{u}}_{1}(0) \cdot \mathbf{u}_{2}(t)\right] \mathrm{d} V, \\
\int_{B} \rho \mathbf{u}_{1} * \overline{\mathbf{b}}_{2} \mathrm{~d} V+\int_{\partial B} g * \mathbf{u}_{1} * \overline{\mathbf{s}}_{2} \mathrm{~d} A=\int_{B} \rho \mathbf{u}_{2} * \overline{\mathbf{b}}_{1} \mathrm{~d} V+\int_{\partial B} g * \mathbf{u}_{2} * \overline{\mathbf{s}}_{1} \mathrm{~d} A,
\end{gathered}
$$

where

$$
\begin{aligned}
\rho \overline{\mathbf{b}}_{\alpha} & =\rho g * \mathbf{b}_{\alpha}+g * \operatorname{div} \mathbf{T}_{\alpha}^{0}+\rho\left(\dot{\mathbf{u}}_{\alpha}(0) t+\mathbf{u}_{\alpha}(0)\right), \\
\mathbf{T}_{\alpha}^{0}(t) & =\int_{-\infty}^{0} \dot{\mathbf{G}}(t-z)\left[\mathbf{E}_{\alpha}(z)\right] \mathrm{d} z, \quad \overline{\mathbf{s}}_{\alpha}=\mathbf{s}_{\alpha}-\mathbf{T}_{\alpha}^{0} \mathbf{n}
\end{aligned}
$$

Proof. We first set

$$
\mathbf{v}(t)=\mathbf{u}_{1}(t), \quad \mathbf{w}(t)=\mathbf{u}_{2}(t),
$$

so that, by mean of the basic equations, from (A7) we get

$$
\begin{aligned}
& \int_{B} \rho\left[\mathbf{u}_{1}(t) \cdot \dot{\mathbf{u}}_{2}(0)+\dot{\mathbf{u}}_{1}(t) \cdot \mathbf{u}_{2}(0)\right] \mathrm{d} V=\int_{B} \rho\left[\mathbf{u}_{1}(0) \cdot \dot{\mathbf{u}}_{2}(t)+\dot{\mathbf{u}}_{1}(0) \cdot \mathbf{u}_{2}(t)\right] \mathrm{d} V \\
& \quad+\int_{0}^{t} \int_{B} \rho\left[\mathbf{u}_{2}(t-s) \cdot \mathbf{b}_{1}(s)-\mathbf{u}_{1}(s) \cdot \mathbf{b}_{2}(t-s)\right] \mathrm{d} V \mathrm{~d} s \\
& \quad+\int_{0}^{t} \int_{\partial B}\left[\mathbf{u}_{2}(t-s) \cdot s_{1}(s)-\mathbf{u}_{1}(s) \cdot \mathbf{s}_{2}(t-s)\right] \mathrm{d} A \mathrm{~d} s \\
& \quad+\int_{0}^{t} \int_{B}\left\{\mathbf{E}_{1}(s) \cdot \int_{-\infty}^{t-s} \mathbf{G}(t-s-z)\left[\dot{\mathbf{E}}_{2}(z)\right] \mathrm{d} z-\mathbf{E}_{2}(t-s)\right. \\
& \left.\quad \cdot \int_{-\infty}^{s} \mathbf{G}(s-z)\left[\dot{\mathbf{E}}_{1}(z)\right] \mathrm{d} z\right\} \mathrm{d} V \mathrm{~d} s .
\end{aligned}
$$


By a straightforward calculation we get successively

$$
\begin{aligned}
\int_{0}^{t} & \int_{B}\left\{\mathbf{E}_{1}(s) \cdot \int_{-\infty}^{t-s} \mathbf{G}(t-s-z)\left[\dot{\mathbf{E}}_{2}(z)\right] \mathrm{d} z-\mathbf{E}_{2}(t-s) \cdot \int_{-\infty}^{s} \mathbf{G}(s-z)\left[\dot{\mathbf{E}}_{1}(z)\right] \mathrm{d} z\right\} \mathrm{d} V \mathrm{~d} s \\
= & \int_{0}^{t} \frac{\mathrm{d}}{\mathrm{d} s}\left\{\int_{-\infty}^{s} \mathrm{~d} z \int_{-\infty}^{t-s} \mathrm{~d} r \int_{B} \mathbf{E}_{1}(z) \cdot \dot{\mathbf{G}}(t-z-r)\left[\mathbf{E}_{2}(r)\right] \mathrm{d} V\right\} \mathrm{d} s \\
= & \int_{\infty}^{t} \mathrm{~d} z \int_{-\infty}^{0} \mathrm{~d} r \int_{B} \mathbf{E}_{1}(z) \cdot \dot{\mathbf{G}}(t-z-r)\left[\mathbf{E}_{2}(r)\right] \mathrm{d} V \\
& -\int_{-\infty}^{0} \mathrm{~d} z \int_{-\infty}^{t} \mathrm{~d} r \int_{B} \mathbf{E}_{1}(z) \cdot \dot{\mathbf{G}}(t-z-r)\left[\mathbf{E}_{2}(r)\right] \mathrm{d} V \\
= & \int_{0}^{t} \int_{B}\left\{\mathbf{E}_{1}(t-s) \cdot \mathbf{T}_{2}^{0}(s)-\mathbf{E}_{2}(t-s) \cdot \mathbf{T}_{1}^{0}(s)\right\} \mathrm{d} V \mathrm{~d} s .
\end{aligned}
$$

If we substitute (6.8) into (6.7) and use the notation of (6.1) we easily get the relation (6.3).

Taking the convolution of relation (6.3) with $g$, we conclude, by means of the divergence theorem and relation (6.5), that the relation (6.4) holds. Thus, the proof is complete.

REMARK. If we use the identity (A4) we get the reciprocal relations established in [4]. Other reciprocal relations may be found by using the identities (A6), (A8) and (A9).

\section{CESÀRO MEANS AND AUTOCORRELATIONS}

In this last section we assume that the relaxation tensor $\mathbf{G}(\cdot)$ is symmetric, $-\dot{\mathbf{G}}(\cdot)$ is of positive type and $\mathbf{G}(\cdot)$ satisfies the condition

$$
\int_{-\infty}^{t} \int_{-\infty}^{t} \dot{\mathbf{A}}(z) \cdot \mathbf{G}(2 t-z-r)[\dot{\mathbf{A}}(r)] \mathrm{d} z \mathrm{~d} r \geqslant \lambda_{0} \mathbf{A}(t) \cdot \mathbf{A}(t), \quad \lambda_{0} \text {-positive constant, }
$$

for every symmetric tensor $\mathbf{A}(\cdot)$ in $C^{1}$ on $(-\infty, \infty)$.

If $\mathbf{A}(\infty)=\mathbf{0}$ and $\dot{\mathbf{G}}(\infty)=\mathbf{0}$, then a straightforward calculation proves that

$$
\begin{aligned}
\int_{-\infty}^{t} \int_{-\infty}^{t} \dot{\mathbf{A}}(z) \cdot \mathbf{G}(2 t-z-r) & {[\dot{\mathbf{A}}(r)] \mathrm{d} z \mathrm{~d} r=\mathbf{A}(t) \cdot \mathbf{G}(\infty)[\mathbf{A}(t)] } \\
& +\int_{-\infty}^{t} \int_{-\infty}^{t}[\mathbf{A}(t)-\mathbf{A}(z)] \cdot \ddot{\mathbf{G}}(2 t-z-r)[\mathbf{A}(t)-\mathbf{A}(r)] \mathrm{d} z \mathrm{~d} r,
\end{aligned}
$$

so that the inequality (7.1) may be satisfied if, for example, $\mathbf{G}(\infty)$ is a positive definite tensor and $\ddot{\mathbf{G}}(\cdot)$ is of positive type.

Let $\left[\mathbf{u}_{\alpha}, \mathbf{E}_{\alpha}, \mathbf{S}_{\alpha}\right], \quad(\alpha=1,2)$, be two viscoelastic processes corresponding to $\left\{\mathbf{G}, \rho, \mathbf{b}_{\alpha} ; \hat{\mathbf{t}}_{\alpha}, \hat{\mathbf{s}}_{\alpha} ;\left[\boldsymbol{\omega}_{\alpha}, \mathbf{R}_{\alpha}, \mathbf{T}_{\alpha}\right]\right\},(\alpha=1,2)$, respectively. Guided by the above results and the results of [22], for each real number $\tau$, we introduce the functions $I_{\alpha \beta}(\tau, \cdot), K_{\alpha \beta}(\tau, \cdot)$, $U_{\alpha \beta}(\tau, \cdot), \mathscr{D}_{\alpha \beta}(\tau, \cdot)$ and $\mathscr{E}_{\alpha \beta}(\tau, \cdot)$, which are defined on $(-\infty, \infty)$ by setting

$$
\begin{gathered}
I_{\alpha \beta}(\tau, t)=\frac{1}{2} \int_{B} \rho \mathbf{u}_{\alpha}(t+\tau) \cdot \mathbf{u}_{\beta}(t) \mathrm{d} V \\
-\frac{1}{2} \int_{0}^{t}\left\{\int_{-\infty}^{s+\tau} d z \int_{-\infty}^{s} \mathrm{~d} r \int_{B} \mathbf{E}_{\alpha}(z) \cdot \dot{\mathbf{G}}(2 s+\tau-z-r)\left[\mathbf{E}_{\beta}(r)\right] \mathrm{d} V\right\} \mathrm{d} s \\
K_{\alpha \beta}(\tau, t)=\frac{1}{2} \int_{B} \rho \dot{u}_{\alpha}(t+\tau) \cdot \dot{\mathbf{u}}_{\beta}(t) \mathrm{d} V \\
U_{\alpha \beta}(\tau, t)=\frac{1}{2} \int_{-\infty}^{t+\tau} \mathrm{d} z \int_{-\infty}^{t} \mathrm{~d} r \int_{B} \dot{\mathbf{E}}_{\alpha}(z) \cdot \mathbf{G}(2 t+\tau-z-r)\left[\dot{\mathbf{E}}_{\beta}(r)\right] \mathrm{d} V \\
\mathscr{D}_{\alpha \beta}(\tau, t)=-\int_{0}^{t}\left\{\int_{-\infty}^{s+\tau} \mathrm{d} z \int_{-\infty}^{s} \mathrm{~d} r \int_{B} \dot{\mathbf{E}}_{\alpha}(z) \cdot \dot{\mathbf{G}}(2 s+\tau-z-r)\left[\dot{\mathbf{E}}_{\beta}(r)\right] \mathrm{d} V\right\} \mathrm{d} s, \\
\mathscr{E}_{\alpha \beta}(\tau, t)=K_{\alpha \beta}(\tau, t)+U_{\alpha \beta}(\tau, t)+\mathscr{D}_{\alpha \beta}(\tau, t) .
\end{gathered}
$$


We shall write

$$
\begin{aligned}
& I_{\alpha}(t)=I_{\alpha \alpha}(0, t), \quad K_{\alpha}(t)=K_{\alpha \alpha}(0, t), \quad U_{\alpha}(t)=U_{\alpha \alpha}(0, t), \\
& \mathscr{D}_{\alpha}(t)=\mathscr{D}_{\alpha \alpha}(0, t), \quad \mathscr{E}_{\alpha}(t)=\mathscr{E}_{\alpha \alpha}(0, t),
\end{aligned}
$$

so that $K_{\alpha}, U_{\alpha}, \mathscr{D}_{\alpha}$ and $\mathscr{E}_{\alpha}$, are, respectively, the kinetic energy, the strain energy, the dissipation energy and the total energy associated with the viscoelastic process $\left[\mathbf{u}_{\alpha}, \mathbf{E}_{\alpha}, \mathbf{S}_{\alpha}\right]$. Then, we introduce the autocorrelations of the velocity field and the strain field by

$$
\lim _{T \rightarrow \infty} \frac{1}{T} \int_{0}^{T} K_{\alpha \beta}(\tau, t) \mathrm{d} t, \quad \lim _{T \rightarrow \infty} \frac{1}{T} \int_{0}^{T} U_{\alpha \beta}(\tau, t) \mathrm{d} t,
$$

and the Cesàro means of the kinetic and strain energies by

$$
\lim _{T \rightarrow \infty} \frac{1}{T} \int_{0}^{T} K_{\alpha}(t) \mathrm{d} t, \quad \lim _{T \rightarrow \infty} \frac{1}{T} \int_{0}^{T} U_{\alpha}(t) \mathrm{d} t .
$$

In what follows we shall show that the above limits exist and are finite. In this aim, we set

$$
\mathbf{v}(t)=\mathbf{u}_{\beta}(t), \quad \mathbf{w}(t)=\mathbf{u}_{\alpha}(t+\tau),
$$

into the identities (A3), (A2) and (A4). As in deduction of the identities (3.7), (3.11) and (3.16), we get the following identities

$$
\begin{aligned}
\frac{\partial \mathscr{E}_{\alpha \beta}}{\partial t}(\tau, t)= & \frac{1}{2} \int_{B} \rho\left[\dot{\mathbf{u}}_{\beta}(t) \cdot \mathbf{b}_{\alpha}(t+\tau)+\dot{\mathbf{u}}_{\alpha}(t+\tau) \cdot \mathbf{b}_{\beta}(t)\right] \mathrm{d} V \\
& \quad+\frac{1}{2} \int_{\partial B}\left[\dot{\mathbf{u}}_{\beta}(t) \cdot \mathbf{s}_{\alpha}(t+\tau)+\dot{\mathbf{u}}_{\alpha}(t+\tau) \cdot \mathbf{s}_{\beta}(t)\right] \mathrm{d} A \\
\frac{\partial^{2} I_{\alpha \beta}}{\partial t^{2}}(\tau, t)= & 2 K_{\alpha \beta}(\tau, t)-2 U_{\alpha \beta}(\tau, t) \\
& \quad+\frac{1}{2} \int_{B} \rho\left[\mathbf{u}_{\beta}(t) \cdot \mathbf{b}_{\alpha}(t+\tau)+\mathbf{u}_{\alpha}(t+\tau) \cdot \mathbf{b}_{\beta}(t)\right] \mathrm{d} V \\
& \quad+\frac{1}{2} \int_{\partial B}\left[\mathbf{u}_{\beta}(t) \cdot \mathbf{s}_{\alpha}(t+\tau)+\mathbf{u}_{\alpha}(t+\tau) \cdot \mathbf{s}_{\beta}(t)\right] \mathrm{d} A \\
\frac{\partial I_{\alpha \beta}}{\partial t}(\tau, t)=- & \frac{1}{2} \int_{-\infty}^{0} \mathrm{~d} r \int_{-\infty}^{2 t+\tau} \mathrm{d} z \int_{B} \mathbf{E}_{\alpha}(z) \cdot \dot{\mathbf{G}}(2 t+\tau-z-r)\left[\mathbf{E}_{\beta}(r)\right] \mathrm{d} V \\
+ & +\frac{1}{2} \int_{B} \rho\left[\mathbf{u}_{\alpha}(2 t+\tau) \cdot \dot{\mathbf{u}}_{\beta}(0)+\dot{\mathbf{u}}_{\alpha}(2 t+\tau) \cdot \mathbf{u}_{\beta}(0)\right] \mathrm{d} V \\
+ & \left.\frac{1}{2} \int_{0}^{t} \int_{B} \rho\left[\mathbf{u}_{\alpha}(t+\tau+s) \cdot \mathbf{b}_{\beta}(t-s)-\mathbf{u}_{\beta}(t-s)\right) \cdot \mathbf{b}_{\alpha}(t+\tau+s)\right] \mathrm{d} V \mathrm{~d} s \\
+ & +\frac{1}{2} \int_{0}^{t} \int_{\partial B}\left[\mathbf{u}_{\alpha}(t+\tau+s) \cdot \mathbf{s}_{\beta}(t-s)-\mathbf{u}_{\beta}(t-s) \cdot \mathbf{s}_{\alpha}(t+\tau+s)\right] \mathrm{d} A \mathrm{~d} s
\end{aligned}
$$

Furthermore, we suppose that the viscoelastic processes $\left[\mathbf{u}_{\alpha}, \mathbf{E}_{\alpha}, \mathbf{S}_{\alpha}\right], \alpha=1,2$, are compatible with the following boundary conditions

$$
\begin{gathered}
\hat{\mathbf{u}}_{\alpha}(\mathbf{x}, t)=\mathbf{0} \text { on } \partial B_{1} \times[0, \infty), \\
\hat{\mathbf{s}}_{\alpha}(\mathbf{x}, t)=\boldsymbol{\Phi}_{\alpha}(\mathbf{x}, t) \mathbf{n}(\mathbf{x}) \quad \text { on } \partial B_{2} \times[0, \infty),
\end{gathered}
$$

where the prescribed fields $\boldsymbol{\Phi}_{\alpha}$ are continuous differentiable on $\bar{B} \times[0, \infty)$. Concerning the external systems $\left\{\mathbf{b}_{\alpha}, \mathbf{s}_{\alpha}\right\}$ we introduce the functions

$$
\begin{gathered}
\varphi_{\alpha}(t)=\left\{2 \int_{B}\left|\boldsymbol{\Phi}_{\alpha}(t)\right|^{2} \mathrm{~d} V\right\}^{1 / 2}, \quad \psi_{\alpha}(t)=\left\{2 \int_{B}\left|\dot{\boldsymbol{\Phi}}_{\alpha}(t)\right|^{2} \mathrm{~d} V\right\}^{1 / 2}, \\
\chi_{\alpha}(t)=\left\{2 \int_{B} \rho\left|\mathbf{b}_{\alpha}(t)+\frac{1}{\rho} \operatorname{div} \boldsymbol{\Phi}_{\alpha}(t)\right|^{2} \mathrm{~d} V\right\}^{1 / 2} .
\end{gathered}
$$


THEOREM 8. Suppose that the viscoelastic processes $\left[\mathbf{u}_{\alpha}, \mathbf{E}_{\alpha}, \mathbf{S}_{\alpha}\right]$ are compatible with the boundary conditions (7.13) and (7.14), and that the following hypothesis holds

$$
\psi_{\alpha}, \chi_{\alpha} \in L^{1}[0, \infty) \text { and } \varphi_{\alpha}(t) \rightarrow 0 \text { as } t \rightarrow \infty .
$$

Then, for each $\tau$, the limits

$$
\begin{aligned}
& \mathscr{D}_{\alpha \beta}(\tau, \infty)=\lim _{t \rightarrow \infty} \mathscr{D}_{\alpha \beta}(\tau, t), \\
& \mathscr{E}_{\alpha \beta}(\tau, \infty)=\lim _{t \rightarrow \infty} \mathscr{E}_{\alpha \beta}(\tau, t),
\end{aligned}
$$

exists and are finite.

Proof. In view of the boundary conditions (7.13) and (7.14), the identity (7.10) can be written in the form

$$
\frac{\partial \mathscr{C}_{\alpha \beta}}{\partial t}(\tau, t)=\frac{\partial \Phi_{\alpha \beta}}{\partial t}(\tau, t)-\Phi_{\alpha \beta}^{\prime}(\tau, t)+\Phi_{\alpha \beta}^{\prime \prime}(\tau, t)
$$

where

$$
\begin{aligned}
& \Phi_{\alpha \beta}(\tau, t)=\frac{1}{2} \int_{B}\left[\Phi_{\alpha}(t+\tau) \cdot \mathbf{E}_{\beta}(t)+\Phi_{\beta}(t) \cdot \mathbf{E}_{\alpha}(t+\tau)\right] \mathrm{d} V, \\
& \Phi_{\alpha \beta}^{\prime}(\tau, t)=\frac{1}{2} \int_{B}\left[\dot{\Phi}_{\alpha}(t+\tau) \cdot \mathbf{E}_{\beta}(t)+\dot{\Phi}_{\beta}(t) \cdot \mathbf{E}_{\alpha}(t+\tau)\right] \mathrm{d} V,
\end{aligned}
$$

$\Phi_{\alpha \beta}^{\prime \prime}(\tau, t)=\frac{1}{2} \int_{B} \rho\left\{\dot{\mathbf{u}}_{\beta}(t) \cdot\left[\mathbf{b}_{\alpha}(t+\tau)+\frac{1}{\rho} \operatorname{div} \Phi_{\alpha}(t+\tau)\right]+\dot{\mathbf{u}}_{\alpha}(t+\tau) \cdot\left[\mathbf{b}_{\beta}(t)+\frac{1}{\rho} \operatorname{div} \Phi_{\beta}(t)\right]\right\} \mathrm{d} V$.

On the basis of the Schwarz's inequality, the relation (7.1) and the inequalities

$$
K_{\alpha} \leqslant \mathscr{E}_{\alpha}, \quad U_{\alpha} \leqslant \mathscr{E}_{\alpha}, \quad \mathscr{D}_{\alpha} \leqslant \mathscr{E}_{\alpha},
$$

we obtain the following estimates

$$
\begin{gathered}
\left|\Phi_{\alpha \beta}(\tau, t)\right| \leqslant \frac{1}{2} \lambda_{0}^{-1 / 2}\left[\varphi_{\alpha}(t+\tau) \mathscr{E}_{\beta}(t)^{1 / 2}+\varphi_{\beta}(t) \mathscr{E}_{\alpha}(t+\tau)^{1 / 2}\right], \\
\left|\Phi_{\alpha \beta}^{\prime}(\tau, t)\right| \leqslant \frac{1}{2} \lambda_{0}^{-1 / 2}\left[\psi_{\alpha}(t+\tau) \mathscr{E}_{\beta}(t)^{1 / 2}+\psi_{\beta}(t) \mathscr{E}_{\alpha}(t+\tau)^{1 / 2}\right], \\
\left|\Phi_{\alpha \beta}^{\prime \prime}(\tau, t)\right| \leqslant \frac{1}{2}\left[\chi_{\alpha}(t+\tau) \mathscr{E}_{\beta}(t)^{1 / 2}+\chi_{\beta}(t) \mathscr{E}_{\alpha}(t+\tau)^{1 / 2}\right] .
\end{gathered}
$$

Let us first prove that the total energy $\mathscr{E}_{\alpha}$ is bounded on $[0, \infty)$. Let $T \in[0, \infty)$ be arbitrary. If we set $\tau=0$ and we make $\alpha=\beta$ in (7.19) and we integrate the result with respect to $t$, then we get

$$
\mathscr{E}_{\alpha}(t)=\mathscr{E}_{\alpha}(0)-\Phi_{\alpha \alpha}(0,0)+\Phi_{\alpha \alpha}(0, t)+\int_{0}^{t}\left[-\Phi_{\alpha \alpha}^{\prime}(0, s)+\Phi_{\alpha \alpha}^{\prime \prime}(0, s)\right] \mathrm{d} s
$$

By estimating the right-hand side of relation (7.27) with the help of relations (7.24)-(7.26), we deduce

$$
\begin{aligned}
\mathscr{E}_{\alpha}(t) \leqslant \mathscr{E}_{\alpha}(0)- & \Phi_{\alpha \alpha}(0,0)+\lambda_{0}^{-1 / 2} \varphi_{\alpha}(t) \mathscr{E}_{\alpha}(t)^{1 / 2} \\
& +\int_{0}^{t}\left[\lambda_{0}^{-1 / 2} \psi_{\alpha}(s)+\chi_{\alpha}(s)\right] \mathscr{E}_{\alpha}(s)^{1 / 2} \mathrm{~d} s \leqslant \mathscr{E}_{\alpha}(0)-\Phi_{\alpha \alpha}(0,0)+C \max _{[0, T]} \mathscr{E}_{\alpha}^{1 / 2},
\end{aligned}
$$

where

$$
C=\lambda_{0}^{-1 / 2} \sup _{[0, \infty)} \varphi_{\alpha}+\int_{0}^{\infty}\left[\lambda_{0}^{-1 / 2} \psi_{\alpha}(s)+\chi_{\alpha}(s)\right] \mathrm{d} s
$$


Thus,

$$
\max _{[0, T]} \mathscr{E}_{\alpha} \leqslant \mathscr{E}_{\alpha}(0)-\Phi_{\alpha \alpha}(0,0)+C \max _{[0, T]} \mathscr{E}_{\alpha}^{1 / 2} \leqslant \mathscr{E}_{\alpha}(0)-\Phi_{\alpha \alpha}(0,0)+\frac{1}{2} C^{2}+\frac{1}{2} \max _{[0, T]} \mathscr{E}_{\alpha},
$$

and, hence,

$$
\max _{[0, T]} \mathscr{E}_{\alpha} \leqslant 2\left[\mathscr{E}_{\alpha}(0)-\Phi_{\alpha \alpha}(0,0)\right]+C^{2}
$$

Because $T \in[0, \infty)$ is arbitrary, it follows that $\mathscr{C}_{\alpha}(t)$ is bounded on $[0, \infty)$.

We now proceed to prove that, for each $\tau$, the limits (7.17) and (7.18) exist and are finite. We first note that $\mathscr{D}_{\alpha}$ is a nondecreasing function on $[0, \infty)$. Because $\mathscr{E}_{\alpha}$ is bounded on $[0, \infty)$, the inequality (7.23) implies that

$$
\mathscr{D}_{\alpha}(\infty)=\lim _{t \rightarrow \infty} \mathscr{D}_{\alpha}(t),
$$

exists and is finite. Therefore, for each $\varepsilon>0$, we can choose $t_{0}=t_{0}(\varepsilon, \alpha)>0$ so that, if $t_{1}^{\prime}$ and $t_{2}^{\prime}$ are any numbers that satisfy $t_{0} \leqslant t_{1}^{\prime} \leqslant t_{2}^{\prime}<\infty$, then we have

$$
\mathscr{D}_{\alpha}\left(t_{2}^{\prime}\right)-\mathscr{D}_{\alpha}\left(t_{1}^{\prime}\right)=-\int_{t_{1}^{\prime}}^{t_{2}}\left\{\int_{-\infty}^{s} \int_{-\infty}^{s} \int_{B} \dot{\mathbf{E}}_{\alpha}(z) \cdot \dot{\mathbf{G}}(2 s-z-r)\left[\dot{\mathbf{E}}_{\alpha}(r)\right] \mathrm{d} V \mathrm{~d} z \mathrm{~d} r\right\} \mathrm{d} s<\varepsilon .
$$

Let $t_{1}$ and $t_{2}$ be two arbitrary numbers so that $t_{0}+|\tau| \leqslant t_{1} \leqslant t_{2}<\infty$. Then the Schwarz inequality and the relation (7.33) lead to

$$
\begin{aligned}
\left|\mathscr{D}_{\alpha \beta}\left(\tau, t_{2}\right)-\mathscr{D}_{\alpha \beta}\left(\tau, t_{1}\right)\right| & \\
= & \left|-\int_{t_{1}}^{t_{2}}\left\{\int_{-\infty}^{s+\tau} \mathrm{d} z \int_{-\infty}^{s} \mathrm{~d} r \int_{B} \dot{\mathbf{E}}_{\alpha}(z) \cdot \dot{\mathbf{G}}(2 s+\tau-z-r)\left[\dot{\mathbf{E}}_{\beta}(r)\right] \mathrm{d} V\right\} \mathrm{d} s\right| \\
\leqslant & \left\{-\int_{t_{1}}^{t_{2}}\left[\int_{-\infty}^{s+\tau} \mathrm{d} z \int_{-\infty}^{s+\tau} \mathrm{d} r \int_{B} \dot{\mathbf{E}}_{\alpha}(z) \cdot \dot{\mathbf{G}}(2 s+2 \tau-z-r)\left[\dot{\mathbf{E}}_{\alpha}(r)\right] \mathrm{d} V\right] \mathrm{d} s\right\}^{1 / 2} \\
& \cdot\left\{-\int_{t_{1}}^{t_{2}}\left[\int_{-\infty}^{s} \mathrm{~d} z \int_{-\infty}^{s} \mathrm{~d} r \int_{B} \dot{\mathbf{E}}_{\beta}(z) \cdot \dot{\mathbf{G}}(2 s-z-r)\left[\dot{\mathbf{E}}_{\beta}(r)\right] \mathrm{d} V\right] \mathrm{d} s\right\}^{1 / 2}<\varepsilon .
\end{aligned}
$$

This proves that the limit in relation (7.17) exists and is finite.

It is now possible for us to prove that, for each $\tau$, the limit (7.18) exists and is finite. Let $\varepsilon>0$ be arbitrary. Since $\mathscr{E}_{\alpha}(t)$ is continuous on $(-\infty, \infty)$ and bounded on $[0, \infty)$, it is bounded on the interval $[-|\tau|, \infty)$ and the hypotheses on $\varphi, \psi$ and $\chi$ ensure that we can choose $t_{0}^{*}=t_{0}^{*}(\varepsilon, \tau, \alpha, \beta)>0$ in such a way that

$$
\begin{gathered}
\lambda_{0}^{-1 / 2}\left[\sup _{[0, \infty)} \mathscr{E}_{\beta}^{1 / 2}\right] \varphi_{\alpha}(t+\tau)+\lambda_{0}^{-1 / 2}\left[\sup _{[-|\tau|, \infty)} \mathscr{E}_{\alpha}^{1 / 2}\right] \varphi_{\beta}(t)<\frac{2}{3} \varepsilon, \quad t_{0}^{*} \leqslant t<\infty, \\
{\left[\sup _{[0, \infty)} \mathscr{E}_{\beta}^{1 / 2}\right] \int_{t_{0}^{*}}^{\infty}\left[\lambda_{0}^{-1 / 2} \psi_{\alpha}(t+\tau)+\chi_{\alpha}(t+\tau)\right] \mathrm{d} t+\left[\sup _{[-|\tau|, \infty)} \mathscr{E}_{\alpha}^{1 / 2}\right] \int_{t^{*}}^{\infty}\left[\lambda_{0}^{-1 / 2} \psi_{\beta}(t)+\chi_{\beta}(t)\right] \mathrm{d} t<\frac{2}{3} \varepsilon .}
\end{gathered}
$$

Let $t_{1} \leqslant t_{2}$ be two numbers in $\left[t_{0}^{*}, \infty\right)$. We integrate both sides of relation (7.19) with respect to $t$, over the interval $\left[t_{1}, t_{2}\right]$. We find

$$
\mathscr{E}_{\alpha \beta}\left(\tau, t_{2}\right)-\mathscr{E}_{\alpha \beta}\left(\tau, t_{1}\right)=\Phi_{\alpha \beta}\left(\tau, t_{2}\right)-\Phi_{\alpha \beta}\left(\tau, t_{1}\right)+\int_{t_{1}}^{t_{2}}\left[-\Phi_{\alpha \beta}^{\prime}(\tau, t)+\Phi_{\alpha \beta}^{\prime \prime}(\tau, t)\right] \mathrm{d} t
$$

By means of the estimates (7.24)-(7.26) and by using the relations (7.35) and (7.36), we get

$$
\begin{aligned}
& \left|\mathscr{E}_{\alpha \beta}\left(\tau, t_{2}\right)-\mathscr{E}_{\alpha \beta}\left(\tau, t_{1}\right)\right| \leqslant \frac{1}{2} \lambda_{0}^{-1 / 2}\left(\sup _{[0, \infty)} \mathscr{E}_{\beta}^{1 / 2}\right)\left[\varphi_{\alpha}\left(t_{1}+\tau\right)+\varphi_{\alpha}\left(t_{2}+\tau\right)\right] \\
& \quad+\frac{1}{2} \lambda_{0}^{-1 / 2}\left(\sup _{|-| \tau \mid, \infty)} \mathscr{C}_{\alpha}^{1 / 2}\right)\left[\varphi_{\beta}\left(t_{1}\right)+\varphi_{\beta}\left(t_{2}\right)\right]+\frac{1}{2}\left(\sup _{[-|\tau|, \infty)} \mathscr{E}_{\alpha}^{1 / 2}\right) \int_{t_{1}}^{t_{2}}\left[\lambda_{0}^{-1 / 2} \psi_{\beta}(t)+\chi_{\beta}(t)\right] \mathrm{d} t \\
& \quad+\frac{1}{2}\left(\sup _{[0, \infty)} \mathscr{C}_{\beta}^{1 / 2}\right) \int_{t_{1}}^{t_{2}}\left[\lambda_{0}^{-1 / 2} \psi_{\alpha}(t+\tau)+\chi_{\alpha}(t+\tau)\right] \mathrm{d} t<\varepsilon .
\end{aligned}
$$

Thus, the limit (7.18) exists and is finite. 
THEOREM 9. Suppose that the viscoelastic processes $\left[\mathbf{u}_{\alpha}, \mathbf{E}_{\alpha}, \mathbf{S}_{\alpha}\right]$ are compatible with the boundary conditions (7.13) and (7.14) and the hypothesis (7.16) holds. Then, for each $\tau$, the autocorrelations of the velocity and strain fields exist and have the common value

$$
\lim _{T \rightarrow \infty} \frac{1}{T} \int_{0}^{T} K_{\alpha \beta}(\tau, t) \mathrm{d} t=\lim _{T \rightarrow \infty} \frac{1}{T} \int_{0}^{T} U_{\alpha \beta}(\tau, t) \mathrm{d} t=\frac{1}{2}\left[\mathscr{E}_{\alpha \beta}(\tau, \infty)-\mathscr{D}_{\alpha \beta}(\tau, \infty)\right] .
$$

Moreover, the Cesàro mean of the kinetic and strain energies exist and have the common value

$$
\lim _{T \rightarrow \infty} \frac{1}{T} \int_{0}^{T} K_{\alpha}(t) \mathrm{d} t=\lim _{T \rightarrow \infty} \frac{1}{T} \int_{0}^{T} U_{\alpha}(t) \mathrm{d} t=\frac{1}{2}\left[\mathscr{E}_{\alpha}(\infty)-\mathscr{D}_{\alpha}(\infty)\right]
$$

Proof. On the basis of the hypothesis (7.1) and the Korn inequality [23], it is possible to determine the positive constant $\lambda$ so that

$$
\frac{1}{2} \int_{B} \rho \mathbf{u}_{\alpha}(t) \cdot \mathbf{u}_{\alpha}(t) \mathrm{d} V \leqslant \lambda U_{\alpha}(t) \leqslant \lambda \mathscr{E}_{\alpha}(t)
$$

Now, let $T>|\tau|$ be arbitrary. If we integrate both sides of relation (7.11) with respect to $t$ over $[0, T]$ and we use the identity (7.12) and the boundary conditions (7.13) and (7.14), then we find

$$
\begin{aligned}
2 \int_{0}^{T}\left[K_{\alpha \beta}(\tau, t)-U_{\alpha \beta}(\tau, t)\right] \mathrm{d} t= & -\frac{\partial I_{\alpha \beta}}{\partial t}(\tau, 0) \\
& +\int_{0}^{T}\left[\Sigma_{\alpha \beta}(\tau, T, t)+\Sigma_{\alpha \beta}^{\prime}(\tau, T, t)\right] \mathrm{d} t+\Delta_{\alpha \beta}(\tau, T),
\end{aligned}
$$

where

$$
\begin{aligned}
& \Sigma_{\alpha \beta}(\tau, T, t)=\frac{1}{2} \int_{B}\left[\mathbf{E}_{\alpha}(T+\tau+t) \cdot \boldsymbol{\Phi}_{\beta}(T-t)-\mathbf{E}_{\beta}(T-t) \cdot \mathbf{\Phi}_{\alpha}(T+\tau+t)\right. \\
& \left.-\mathbf{E}_{\alpha}(t+\tau) \cdot \boldsymbol{\Phi}_{\beta}(t)-\mathbf{E}_{\beta}(t) \cdot \boldsymbol{\Phi}_{\alpha}(t+\tau)\right] \mathrm{d} V, \\
& \Sigma_{\alpha \beta}^{\prime}(\tau, T, t)=\frac{1}{2} \int_{B}\left\{\rho \mathbf{u}_{\alpha}(T+\tau+t) \cdot\left[\mathbf{b}_{\beta}(T-t)+\frac{1}{\rho} \operatorname{div} \boldsymbol{\Phi}_{\beta}(T-t)\right]\right. \\
& -\rho \mathbf{u}_{\beta}(T-t) \cdot\left[\mathbf{b}_{\alpha}(T+\tau+t)+\frac{1}{\rho} \operatorname{div} \mathbf{\Phi}_{\alpha}(T+\tau+t)\right] \\
& -\rho \mathbf{u}_{\alpha}(t+\tau) \cdot\left[\mathbf{b}_{\beta}(t)+\frac{1}{\rho} \operatorname{div} \boldsymbol{\Phi}_{\beta}(t)\right] \\
& \left.-\rho \mathbf{u}_{\beta}(t) \cdot\left[\mathbf{b}_{\alpha}(t+\tau)+\frac{1}{\rho} \operatorname{div} \boldsymbol{\Phi}_{\alpha}(t+\tau)\right]\right\} \mathrm{d} V . \\
& \Delta_{\alpha \beta}(\tau, T)=\frac{1}{2} \int_{B} \mathbf{E}_{\alpha}(2 T+\tau) \cdot \int_{-\infty}^{0} \mathbf{G}(-r)\left[\mathbf{E}_{\beta}(r)\right] \mathrm{d} r \mathrm{~d} V \\
& -\frac{1}{2} \int_{-\infty}^{0} \mathrm{~d} r \int_{-\infty}^{0} \mathrm{~d} z \int_{B} \dot{\mathbf{E}}_{\alpha}(2 T+\tau+z) \cdot \mathbf{G}(-z-r)\left[\mathbf{E}_{\beta}(r)\right] \mathrm{d} V \\
& +\frac{1}{2} \int_{B} \rho\left[\mathbf{u}_{\alpha}(2 T+\tau) \cdot \dot{\mathbf{u}}_{\beta}(0)+\dot{\mathbf{u}}_{\alpha}(2 T+\tau) \cdot \mathbf{u}_{\beta}(0)\right] \mathrm{d} V .
\end{aligned}
$$

By using the Schwarz inequality and the relations (7.1), (7.23) and (7.41), we obtain the 
following estimates

$$
\begin{aligned}
\left|\Sigma_{\alpha \beta}(\tau, T, t)\right| \leqslant & \frac{1}{2} \lambda_{0}^{-1 / 2}\left[\mathscr{E}_{\alpha}(T+\tau+t)^{1 / 2} \varphi_{\beta}(T-t)+\mathscr{E}_{\beta}(T-t)^{1 / 2} \varphi_{\alpha}(T+\tau+t)\right. \\
& \left.+\mathscr{E}_{\alpha}(\tau+t)^{1 / 2} \varphi_{\beta}(t)+\mathscr{E}_{\beta}(t)^{1 / 2} \varphi_{\alpha}(\tau+t)\right] \\
\left|\Sigma_{\alpha \beta}^{\prime}(\tau, T, t)\right| \leqslant & \frac{1}{2} \lambda^{1 / 2}\left[\mathscr{E}_{\alpha}(T+\tau+t)^{1 / 2} \chi_{\beta}(T-t)+\mathscr{E}_{\beta}(T-t)^{1 / 2} \chi_{\alpha}(T+\tau+t)\right. \\
& \left.+\mathscr{E}_{\alpha}(\tau+t)^{1 / 2} \chi_{\beta}(t)+\mathscr{E}_{\beta}(t)^{1 / 2} \chi_{\alpha}(\tau+t)\right] \\
\left|\Delta_{\alpha \beta}(\tau, T)\right| \leqslant & \mathscr{E}_{\alpha}(2 T+\tau)^{1 / 2}\left\{\lambda_{\theta}^{-1 / 2}\left(\frac{1}{2} \int_{B}\left|\int_{-\infty}^{0} \mathbf{G}(-r)\left[\mathbf{E}_{\beta}(r)\right] \mathrm{d} r\right|^{2} \mathrm{~d} V\right)^{1 / 2}\right. \\
& +\left(\frac{1}{2} \int_{-\infty}^{0} \int_{-\infty}^{0} \int_{B} \mathbf{E}_{\beta}(z) \cdot \mathbf{G}(-z-r)\left[\mathbf{E}_{\beta}(r)\right] \mathrm{d} V \mathrm{~d} z \mathrm{~d} r\right)^{1 / 2} \\
& \left.+\lambda^{1 / 2}\left(\frac{1}{2} \int_{B} \rho\left|\dot{\mathbf{u}}_{\beta}(0)\right|^{2} \mathrm{~d} V\right)^{1 / 2}+\left(\frac{1}{2} \int_{B} \rho\left|\mathbf{u}_{\beta}(0)\right|^{2} \mathrm{~d} V\right)^{1 / 2}\right\}
\end{aligned}
$$

We now use the estimates $(7.46)-(7.48)$ in the relation $(7.42)$ in order to obtain

$$
\begin{aligned}
& 2\left|\int_{0}^{T}\left[K_{\alpha \beta}(\tau, t)-U_{\alpha \beta}(\tau, t)\right] \mathrm{d} t\right| \leqslant\left|\frac{\partial I_{\alpha \beta}}{\partial t}(\tau, 0)\right| \\
& \quad+\frac{1}{2} \int_{0}^{T}\left\{\mathscr{C}_{\alpha}(T+\tau+t)^{1 / 2}\left[\lambda_{0}^{-1 / 2} \varphi_{\beta}(T-t)+\lambda^{1 / 2} \chi_{\beta}(T-t)\right]\right. \\
& \quad+\mathscr{E}_{\beta}(T-t)^{1 / 2}\left[\lambda_{0}^{-1 / 2} \varphi_{\alpha}(T+\tau+t)+\lambda^{1 / 2} \chi_{\alpha}(T+\tau+t)\right] \\
& \quad+\mathscr{E}_{\alpha}(\tau+t)^{1 / 2} \cdot\left[\lambda_{0}^{-1 / 2} \varphi_{\beta}(t)+\lambda^{1 / 2} \chi_{\beta}(t)\right] \\
& \left.\quad+\mathscr{E}_{\beta}(t)^{1 / 2}\left[\lambda_{0}^{-1 / 2} \varphi_{\alpha}(\tau+t)+\lambda^{1 / 2} \chi_{\alpha}(\tau+t)\right]\right\} \mathrm{d} t \\
& \quad+\mathscr{E}_{\alpha}(2 T+\tau)^{1 / 2}\left\{\lambda_{0}^{-1 / 2}\left(\frac{1}{2} \int_{B}\left|\int_{-\infty}^{0} \mathbf{G}(-r)\left[\mathbf{E}_{\beta}(r)\right] \mathrm{d} r\right|^{2} \mathrm{~d} V\right)^{1 / 2}\right. \\
& \quad+\left(\frac{1}{2} \int_{-\infty}^{0} \int_{-\infty}^{0} \int_{B} \mathbf{E}_{\beta}(z) \cdot \mathbf{G}(-z-r)\left[E_{\beta}(r)\right] \mathrm{d} V\right)^{1 / 2} \\
& \left.\quad+\lambda^{1 / 2}\left(\frac{1}{2} \int_{B} \rho\left|\dot{\mathbf{u}}_{\beta}(0)\right|^{2} \mathrm{~d} V\right)^{1 / 2}+\left(\frac{1}{2} \int_{B} \rho\left|\mathbf{u}_{\beta}(0)\right|^{2} \mathrm{~d} V\right)^{1 / 2}\right\}
\end{aligned}
$$

Further, we divide relation (7.49) by $T$ and let $T \rightarrow \infty$. Because $\mathscr{E}_{\alpha}$ is bounded, by means of the relations $(7.16)$, we deduce

$$
\lim _{T \rightarrow \infty} \frac{1}{T} \int_{0}^{T} K_{\alpha \beta}(\tau, t) \mathrm{d} t=\lim _{T \rightarrow \infty} \frac{1}{T} \int_{0}^{T} U_{\alpha \beta}(\tau, t) \mathrm{d} t .
$$

We proceed now to prove the relation (7.39). To do this, we shall use the relations (7.17), (7.18) and (7.50). We first note that the relation (7.17) implies

$$
\lim _{T \rightarrow \infty} \frac{1}{T} \int_{0}^{T} \mathscr{D}_{\alpha \beta}(\tau, t) \mathrm{d} t=\mathscr{D}_{\alpha \beta}(\tau, \infty) .
$$

Then, for every $T>0$, we have

$$
\begin{aligned}
\mid \frac{1}{T} \int_{0}^{T} K_{\alpha \beta}(\tau, t) \mathrm{d} t-\frac{1}{2}\left[\mathscr{E}_{\alpha \beta}(\tau, \infty)-\mathscr{D}_{\alpha \beta}(\tau, \infty) \mid\right. \\
=\mid \frac{1}{T} \int_{0}^{T}\left\{\frac{1}{2}\left[\mathscr{E}_{\alpha \beta}(\tau, t)-\mathscr{E}_{\alpha \beta}(\tau, \infty)\right]+\frac{1}{2}\left[K_{\alpha \beta}(\tau, t)-U_{\alpha \beta}(\tau, t)\right]\right. \\
\left.-\frac{1}{2}\left[\mathscr{D}_{\alpha \beta}(\tau, t)-\mathscr{D}_{\alpha \beta}(\tau, \infty)\right]\right\} \mathrm{d} t \mid
\end{aligned}
$$




$$
\begin{aligned}
\leqslant & \frac{1}{2 T} \int_{0}^{T}\left|\mathscr{E}_{\alpha \beta}(\tau, t)-\mathscr{E}_{\alpha \beta}(\tau, \infty)\right| \mathrm{d} t+\frac{1}{2 T} \int_{0}^{T}\left|\mathscr{D}_{\alpha \beta}(\tau, t)-\mathscr{D}_{\alpha \beta}(\tau, \infty)\right| \mathrm{d} t \\
& +\frac{1}{2 T}\left|\int_{0}^{T}\left[K_{\alpha \beta}(\tau, t)-U_{\alpha \beta}(\tau, t)\right] \mathrm{d} t\right| .
\end{aligned}
$$

Let $\varepsilon>0$ be an arbitrary number. We choose $T_{0}=T_{0}(\varepsilon, \tau, \alpha, \beta)>0$ so large that

$$
\left|\mathscr{E}_{\alpha \beta}(\tau, t)-\mathscr{E}_{\alpha \beta}(\tau, \infty)\right|<\varepsilon, \quad\left|\mathscr{D}_{\alpha \beta}(\tau, t)-\mathscr{D}_{\alpha \beta}(\tau, \infty)\right|<\varepsilon, \quad t>T_{0} .
$$

Then, we find that

$$
\begin{aligned}
& \left|\frac{1}{T} \int_{0}^{T} K_{\alpha \beta}(\tau, t) \mathrm{d} t-\frac{1}{2}\left[\mathscr{E}_{\alpha \beta}(\tau, \infty)-\mathscr{D}_{\alpha \beta}(\tau, \infty)\right]\right| \leqslant \frac{1}{2 T}\left(\int_{0}^{T_{0}}+\int_{T_{0}}^{T}\right)\left|\mathscr{E}_{\alpha \beta}(\tau, t)-\mathscr{E}_{\alpha \beta}(\tau, \infty)\right| \mathrm{d} t \\
& \quad+\frac{1}{2 T}\left(\int_{0}^{T_{0}}+\int_{T_{0}}^{T}\right)\left|\mathscr{D}_{\alpha \beta}(\tau, t)-\mathscr{D}_{\alpha \beta}(\tau, \infty)\right| \mathrm{d} t+\frac{1}{2 T}\left|\int_{0}^{T}\left[K_{\alpha \beta}(\tau, t)-U_{\alpha \beta}(\tau, t)\right] \mathrm{d} t\right| \\
& \quad \leqslant \frac{1}{2 T} \int_{0}^{T_{0}}\left|\mathscr{C}_{\alpha \beta}(\tau, t)-\mathscr{E}_{\alpha \beta}(\tau, \infty)\right| \mathrm{d} t+\frac{1}{2} \varepsilon+\frac{1}{2 T} \int_{0}^{T_{0}}\left|\mathscr{D}_{\alpha \beta}(\tau, t)-\mathscr{D}_{\alpha \beta}(\tau, \infty)\right| \mathrm{d} t \\
& \quad+\frac{1}{2} \varepsilon+\frac{1}{2 T}\left|\int_{0}^{T}\left[K_{\alpha \beta}(\tau, t)-U_{\alpha \beta}(\tau, t)\right] \mathrm{d} t\right|
\end{aligned}
$$

for every $T>T_{0}$. Therefore,

$$
\limsup _{T \rightarrow \infty}\left|\frac{1}{T} \int_{0}^{T} K_{\alpha \beta}(\tau, t) \mathrm{d} t-\frac{1}{2}\left[\mathscr{E}_{\alpha \beta}(\tau, \infty)-\mathscr{D}_{\alpha \beta}(\tau, \infty)\right]\right|<\varepsilon,
$$

for every $\varepsilon>0$, so that

$$
\lim _{\tau \rightarrow \infty} \frac{1}{T} \int_{0}^{T} K_{\alpha \beta}(\tau, t) \mathrm{d} t=\frac{1}{2}\left[\mathscr{E}_{\alpha \beta}(\tau, \infty)-\mathscr{D}_{\alpha \beta}(\tau, \infty)\right] .
$$

By taking into account the relations (7.50) and (7.56) we deduce the relation (7.39). The relation (7.40) is a direct consequence of the relation (7.39). Thus, the proof is complete.

Remark. The relation (7.50) generalizes the one obtained in [24] for a homogeneous viscoelastic filament.

Acknowledgements-One of the authors (S.C.) acknowledges the warm hospitality extended to him by the G.N.F.M. of the Italian National Research Council and by the Dipartimento di Matematica ed Applicazioni $R$. Caccioppoli during a recent Visiting Professorship. S. Rionero acknowledges the G.N.F.M., the M.P.I. $40 \%$ and $60 \%$ contracts and S.P.A.I.M. of C.N.R. It is a pleasure to record helpful discussions with Professor R. J. Knops.

\section{REFERENCES}

[1] L. BRUN, J. Méc. 8, 167 (1969).

[2] N. S. WILKES, J. Méc. 17, 717 (1978).

3] M. E. GURTIN and I. HERRERA, Q. Appl. Math. 23, 235 (1965)

[4] S. RIONERO and S. CHIRITĂ, Int. J. Engng Sci. 27, 1023 (1989).

[5] N. S. WILKES, Q. J. Mech. Appl. Math. 30, 209 (1977).

[6] D. IEŞAN, Mem. Acad. Sci. Torino Cl. Sci. Fis. Mat. Nat. Ser. 4, 17 (1974).

[7] M. J. LEITMAN and G. M. C. FISHER, The linear theory of viscoelasticity. In Handbuch der Physik (Edited by C. TRUESDELL), Vol. VIa/3. Springer, Berlin (1973).

[8] W. A. DAY, Arch. Rat. Mech. Anal. 40, 155 (1971).

[9] C. M. DAFERMOS, J. Diff. Equations 7, 554 (1970).

[10] C. M. DAFERMOS, Arch. Rat. Mech. Anal. 37, 297 (1970).

[11] A. J. STAVERMAN and F. SCHWARZL, Verh. Akad. Can Wet. Amsterdam B 55, 474 (1952)

[12] D. BLAND, Theory of Linear Viscoelasticity. Pergamon Press, Oxford (1960).

[13] S. C. HUNTER, J. Mech. Phys. Solids 9, 39 (1961).

[14] S. BREUER and E. T. ONAT, ZAMP 15, 184 (1964).

[15] R. M. CHRISTENSEN and P. M. NAGHDI, Acta Mech. 3, 1 (1967).

[16] A. C. ERINGEN, Mechanics of Continua, Chap. 9. Wiley, New York (1967).

[17] E. H. DILL, Simple materials with fading memory. In Continuum Physics, Vol. II: Continuum Mechanics of Single-Substance Bodies, (Edited by A. C. ERINGEN), Chap. 4, p. 289. Academic Press, New York (1975). 
[18] R. J. KNOPS and L. E. PAYNE, Arch. Rat. Mech. Anal. 27, 349 (1968).

[19] H. BREZIS, Operateurs Maximaux Monotones et Semi-Groupes de Contractions dans les Espaces de Hilbert. North-Holland, Amsterdam (1973).

[20] R. J. KNOPS and L. E. PAYNE, Proc. Cambridge Phil. Soc. 66, 481 (1969).

[21] R. J. KNOPS and L .E. PAYNE, Math. Proc. Cambridge Phil. Soc. 103, 535 (1988).

(22] W. A. DAY, Arch. Rat. Mech. Anal. 73, 243 (1980).

[23] G. FICHERA, Existence theorems in elasticity. In Handbuch der Physik (Edited by C. TRUESDELL), Vol. VIa/2. Springer, Berlin (1972).

[24] D. W. REYNOLDS, Q. Appl. Math. 62, 325 (1984).

[25] L. TARTAR, Nonlinear partial differential equations using compactness method. MRC Technical Summary Report No. 1584, University of Wisconsin (1976).

(Received 24 January 1991; accepted 15 March 1991)

\section{APPENDIX}

\section{The Lagrange Identities}

It is not our intention to present an exhaustive description of the class of Lagrange identities but instead we confine ourselves to a sketch of some of types which we will use in this paper.

Thus, we consider a real Hilbert space $H$ endowed with an appropriate inner product $\langle\cdot, \cdot\rangle$. Let $\mathbf{v}$ and $\mathbf{w}$ be arbitrary functions of class $C^{2}$ on $(-\infty, \infty)$ whose values are contained into $H$.

We start with the basic Lagrange identity

$$
\begin{aligned}
\langle\mathbf{v}(t), \dot{\mathbf{w}}(t)\rangle-\langle\dot{\mathbf{v}}(t), \mathbf{w}(t)\rangle= & \langle\mathbf{v}(0), \dot{\mathbf{w}}(0)\rangle-\langle\dot{\mathbf{v}}(0), \mathbf{w}(0)\rangle \\
& +\int_{0}^{t}[\langle\mathbf{v}(s), \ddot{\mathbf{w}}(s)\rangle-\langle\ddot{\mathbf{v}}(s), \mathbf{w}(s)\rangle] \mathrm{d} s,
\end{aligned}
$$

where the superposed dot denotes the derivative with respect to the displayed time variable.

It is easy to see that the above identity may be written in the form

$$
\langle\mathbf{v}(t), \dot{\mathbf{w}}(t)\rangle=\langle\mathbf{v}(0), \dot{\mathbf{w}}(0)\rangle+\int_{0}^{t}[\langle\mathbf{v}(s), \ddot{\mathbf{w}}(s)\rangle+\langle\dot{\mathbf{v}}(s), \dot{\mathbf{w}}(s)\rangle] \mathrm{d} s .
$$

Further, we change $\mathbf{v}(s)$ in $\dot{v}(s)$ into (A2) in order to deduce

$$
\langle\dot{\mathbf{v}}(t), \dot{\mathbf{w}}(t)\rangle=\langle\dot{\mathbf{v}}(0), \dot{\mathbf{w}}(0)\rangle+\int_{0}^{t}[\langle\dot{\mathbf{v}}(s), \ddot{\mathbf{w}}(s)\rangle+\langle\ddot{\mathbf{v}}(s), \dot{\mathbf{w}}(s)\rangle] \mathrm{d} s .
$$

Special choices of the fields $\mathbf{v}$ and $\mathbf{w}$ in the above identities will lead to other particular forms of the basic Lagrange identity. 'Thus, for fixed $t$, we make the change $v(s) \rightarrow \mathbf{v}(t-s)$ and $w(s) \rightarrow w(t+s)$ into (A1)-(A3) in order to obtain

$$
\begin{aligned}
&\langle\mathbf{v}(t), \dot{\mathbf{w}}(t)\rangle+\langle\dot{\mathbf{v}}(t), \mathbf{w}(t)\rangle=\langle\mathbf{v}(0), \dot{\mathbf{w}}(2 t)\rangle+\langle\dot{\mathbf{v}}(0), \mathbf{w}(2 t)\rangle \\
&+\int_{0}^{t}[\langle\ddot{\mathbf{v}}(t-s), \mathbf{w}(t+s)\rangle-\langle\mathbf{v}(t-s), \ddot{\mathbf{w}}(t+s)] \mathrm{d} s, \\
&\langle\mathbf{v}(t), \dot{\mathbf{w}}(t)\rangle=\langle\mathbf{v}(0), \dot{\mathbf{w}}(2 t)\rangle++\int_{0}^{t}[\langle\dot{\mathbf{v}}(t-s), \dot{\mathbf{w}}(t+s)\rangle-\langle\mathbf{v}(t-s), \ddot{\mathbf{w}}(t+s)\rangle] \mathrm{d} s, \\
&\langle\dot{\mathbf{v}}(t), \dot{\mathbf{w}}(t)\rangle=\langle\mathbf{v}(0), \dot{\mathbf{w}}(2 t)\rangle+\int_{0}^{t}[\langle\ddot{\mathbf{v}}(t-s), \dot{\mathbf{w}}(t+s)\rangle-\langle\dot{\mathbf{v}}(t-s), \ddot{\mathbf{w}}(t+s)\rangle] \mathrm{d} s .
\end{aligned}
$$

Finally, we set $\mathbf{v}(s) \rightarrow \mathbf{v}(s)$ and $\mathbf{w}(s) \rightarrow \mathbf{w}(t-s)$ so that we get

$$
\begin{aligned}
\langle\mathbf{v}(t), \dot{w}(0)\rangle+ & \langle\dot{\mathbf{v}}(t), \mathbf{w}(0)\rangle=\langle\mathbf{v}(0), \dot{\mathbf{w}}(t)\rangle+\langle\dot{\mathbf{v}}(0), \mathbf{w}(t)\rangle+\int_{0}^{t}[\langle\ddot{v}(s), \mathbf{w}(t-s)\rangle-\langle\mathbf{v}(s), \ddot{\mathbf{w}}(t-s)\rangle] \mathrm{d} s, \\
\langle\mathbf{v}(t), \dot{w}(0)\rangle & =\langle\mathbf{v}(0), \dot{\mathbf{w}}(t)\rangle+\int_{0}^{t}[\langle\dot{v}(s), \dot{\mathbf{w}}(t-s)\rangle-\langle\mathbf{v}(s), \ddot{\mathbf{w}}(t-s)\rangle] \mathrm{d} s, \\
\langle\dot{\mathbf{v}}(t), \dot{\mathbf{w}}(0)\rangle & =\langle\dot{\mathbf{v}}(0), \dot{\mathbf{w}}(t)\rangle+\int_{0}^{t}[\langle\ddot{\mathbf{v}}(s), \dot{\mathbf{w}}(t-s)\rangle-\langle\dot{\mathbf{v}}(s), \ddot{\mathbf{w}}(t-s)\rangle] \mathrm{d} s .
\end{aligned}
$$

Other forms of the basic Lagrange identity are presented by Tartar [25]. 